\title{
An Efficient Mixed-Mode Representation of Sparse Tensors
}

\author{
Israt Nisa \\ Ohio State University \\ nisa.1@osu.edu \\ Prasant Singh Rawat \\ Ohio State University \\ rawat.15@osu.edu
}

\author{
Jiajia Li \\ Pacific Northwest National \\ Laboratory \\ Jiajia.Li@pnnl.gov
Sriram Krishnamoorthy
Pacific Northwest National
Laboratory
sriram@pnnl.gov

\author{
Aravind Sukumaran-Rajam \\ Ohio State University \\ sukumaranrajam.1@osu.edu
}

\author{
P. Sadayappan \\ University of Utah \\ saday@cs.utah.edu
}

\begin{abstract}
The Compressed Sparse Fiber (CSF) representation for sparse tensors is a generalization of the Compressed Sparse Row (CSR) format for sparse matrices. For a tensor with $d$ modes, typical tensor methods such as CANDECOMP/PARAFAC decomposition (CPD) require a sequence of $d$ tensor computations, where efficient memory access with respect to different modes is required for each of them. The straightforward solution is to use $d$ distinct representations of the tensor, with each one being efficient for one of the $d$ computations. However, a $d$-fold space overhead is often unacceptable in practice, especially with memory-constrained GPUs. In this paper, we present a mixed-mode tensor representation that partitions the tensor's nonzero elements into disjoint sections, each of which is compressed to create fibers along a different mode. Experimental results demonstrate that better performance can be achieved while utilizing only a small fraction of the space required to keep $d$ distinct CSF representations.
\end{abstract}

\section{CCS CONCEPTS}

- Theory of computation $\rightarrow$ Parallel algorithms;

\section{KEYWORDS}

Sparse tensors, MTTKRP, GPU, CANDECOMP/PARAFAC decomposition

\section{ACM Reference Format:}

Israt Nisa, Jiajia Li, Aravind Sukumaran-Rajam, Prasant Singh Rawat, Sriram Krishnamoorthy, and P. Sadayappan. 2019. An Efficient Mixed-Mode Representation of Sparse Tensors. In The International Conference for High Performance Computing, Networking, Storage, and Analysis (SC '19), November 17-22, 2019, Denver, CO, USA. ACM, New York, NY, USA, 13 pages. https://doi.org/10.1145/3295500.3356216

Permission to make digital or hard copies of all or part of this work for personal or classroom use is granted without fee provided that copies are not made or distributed for profit or commercial advantage and that copies bear this notice and the full citation on the first page. Copyrights for components of this work owned by others than ACM must be honored. Abstracting with credit is permitted. To copy otherwise, or republish, to post on servers or to redistribute to lists, requires prior specific permission and/or a fee. Request permissions from permissions@acm.org.

SC '19, November 17-22, 2019, Denver, CO, USA

(C) 2019 Association for Computing Machinery.

ACM ISBN 978-1-4503-6229-0/19/11...\$15.00

https://doi.org/10.1145/3295500.3356216

\section{INTRODUCTION}

Tensors are multidimensional data commonly used in machine learning [2], text analysis [4], healthcare analytics [16], [17], telecommunications [36], [37], and numerous other applications. Tensors are useful because they provide a generalization of storing data for arbitrary number of dimensions, where each dimension is termed a mode. Real world tensors are extremely large and sparse, with high irregularity in shape and distribution of nonzeros. Unlike their dense counterparts, sparse tensors need a compressed storage format to be space efficient.

There exists a vast research history on efficiently representing sparse matrices, which are special tensors with two modes. A natural way of representing sparse matrices is to just store the indices for the non-zero elements, along with its value. One can further optimize the storage by reusing the same row pointer for all the non-zeros in the same row. This format is called Compressed Sparse Row (CSR), and is universally regarded as the de facto representation for sparse matrices. For hyper-sparse matrices with many empty rows, Doubly Compressed Sparse Row (DCSR) format [10] further compresses CSR by storing the row pointers for only the non-empty rows. Compressed Sparse Fiber (CSF) is a generalization of CSR (or DCSR) for higher dimensional tensors.

A full iteration of CANDECOMP/PARAFAC decomposition (CPD) or Tucker Decomposition requires performing Matricized Tensor Times Khatri-Rao Products (MTTKRP), or Tensor-Times-Matrix products (TTM) on every mode. Therefore, many state-of-the-art tensor factorization frameworks create a compact representation of a tensor at each mode to achieve an overall high performance. For illustration, consider an application that performs sparse matrixvector multiplication (SpMV), $y=A x$, in tandem with sparse matrixtranspose-vector multiplication (SpMTV), $z=A^{T} x$. If $A$ is stored in CSR format, then parallelism can be achieved across rows while computing $y=A x$. However, computing $z=A^{T} x$ with CSR would require explicit locks or atomic operations to update $z$. Similarly, storing $A$ in Compressed Sparse Column (CSC) format will achieve parallelism for $z=A^{T} x$, but introduce atomics for $y=A x$. Explicit synchronization is usually prohibitively expensive on multiple architectures, including GPUs. A naïve solution to this conundrum is to store $A$ in both CSR and CSC formats. The same logic extends to tensors: to achieve parallelism and efficient accesses across $d$ modes, $d$ representations of the tensor are maintained. Clearly, the storage overhead will increase with the number of modes, making this solution impractical for higher order tensors. 
This paper attempts to reconcile two conflicting objectives: reducing the overall storage overhead for tensors by using a single representation for all the modes, and achieving equal or better performance compared to the naïve approach of storing $d$ representations. A previous effort in this direction by Smith et al. was proposed in the SPLATT library [32] - given $d$ CSF representations for $d$ modes, their implementation selects the CSF where the shortest dimension is at the outermost level as the only representative. Computation on all the modes will use the same representative CSF. We term this storage method SPLATT-ONE, in contrast to SPLATT-ALL, which represents the strategy of creating a different CSF representation for each mode. Even though selecting one out of $d$ CSF representations is an easy technique to reduce storage overhead, often further analysis in identifying the sparsity structure of the real-world tensors can further improve both parallelism and compression.

In this paper, we propose a novel mixed-mode format termed MM-CSF, where long fibers ${ }^{1}$ on each mode are first identified and then stored on their most suitable modes. Doing so achieves better compression, which not only reduces space requirement, but also provides performance improvement. Revisiting the illustrative example, while performing SpMV (SpMTV) with the mixed-mode format, the nonzeros in the CSR (CSC) representation can exploit the parallelism and compression in the long rows (columns), and the rest of the nonzeros in the CSC (CSR) representation will require atomics. Figure 1 further elucidates our insight behind mixed-mode. The matrix in the figure is sparse, with only one non-empty row and column. Using either CSR or CSC representation would require storing 38 elements in row pointers, column indices, and nonzeros; simultaneously maintaining both CSR and CSC representations for efficient computation would require storing 76 elements. In contrast, the mixed-mode format will store the dense row in CSR format, and the dense column in CSC format, reducing the overall storage to 32 elements. Furthermore, as illustrated later in the paper, mixed-mode storage incurs fewer global memory transactions due to better compression when compared to SPLATT-ONE, and uncovers a scope for finer grained parallelism when compared to SPLATT-ALL. In summary, this work makes the following contributions.

- It proposes MM-CSF, a novel storage format that exploits the underlying sparsity structure of real-world tensors, and provides compact storage without compromising on computational efficiency of tensor operations.

- It provides experimental data demonstrating that compared to the storage formats used in the state-of-the-art CPU frameworks, MM-CSF can save up to $75 \%$ space, while improving MTTKRP performance by up to $63 \times$.

- On a NVIDIA Volta GPU, it demonstrates that MM-CSF outperforms the state-of-the-art GPU storage format, BCSF [26], by up to a factor of $2 \times$ in computing MTTKRP, and reduces up to $85 \%$ of the space requirement.

The rest of the paper is organized as follows. Section 2 describes the tensor notations and gives an overview of tensor decomposition. Section 3 gives a brief overview of the sparse tensor storage formats used by the state-of-the-art tensor factorization frameworks.

${ }^{1}$ Generalization of matrix rows and columns, created by holding all but one index constant.

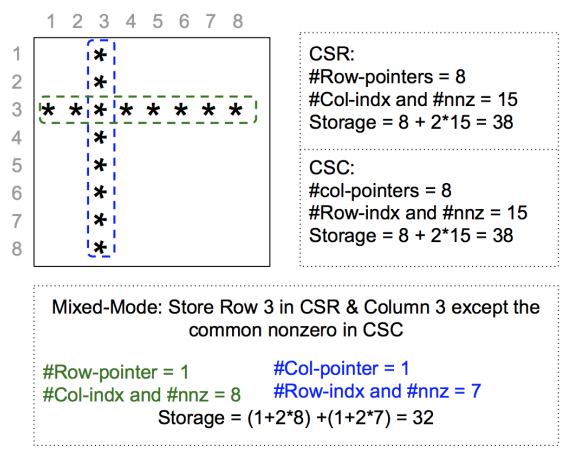

Figure 1: Scope of compression using mixed-mode representation in a matrix

\begin{tabular}{c|l}
\hline Terminologies & Description \\
\hline $\mathcal{X}$ & Tensor \\
$d$ & Tensor order \\
$M$ & Number of nonzeros in $\mathcal{X}$ \\
$S_{n}$ & Number of slices in $\mathcal{X}$ on mode- $n$ \\
$F_{n}$ & Number of fibers in $\mathcal{X}$ on mode- $n$ \\
$F_{B-n}$ & Number of fibers in BCSF on mode- $n$ \\
$A, B, C$ & Factor matrices \\
$R$ & Tensor rank \\
\hline CSF- $n$ & CSF representation of $\mathcal{X}$ on mode- $n$ \\
${ }^{*}$-ALL & Implementation with $d$ different CSF representations of $\mathcal{X}$ \\
${ }^{*}$-ONE & Implementation with one CSF representation of $\mathcal{X}$ \\
MM-CSF & Mixed-mode single CSF representation of $\mathcal{X}$ \\
\hline
\end{tabular}

Table 1: Tensor notations

Sections 4 and 5 discuss our proposed MM-CSF representation in detail and describe the acceleration of MTTKRP computation on GPUs with the MM-CSF representation. Section 6 presents experimental evaluation, Section 7 discusses the related work, and Section 8 concludes.

\section{TENSOR BACKGROUND}

\subsection{Tensor Notation}

The tensor notations used in this paper are adapted from Kolda and Sun [21]. We represent tensors as $\mathcal{X}$. Let us assume that $\mathcal{X}$ is a third-order tensor of dimension $I \times J \times K$, and the rank of the tensor is $R$. The dimensions of a tensor are also known as modes. This third-order tensor $X$ can be decomposed into three factor matrices, $A \in \mathbb{R}^{I \times R}, B \in \mathbb{R}^{J \times R}$, and $C \in \mathbb{R}^{K \times R}$. The nonzero elements of $\mathcal{X}$ can be represented as a list of coordinates, $\langle i, j, k, v a l s\rangle$. Individual elements of $\mathcal{X}$ are expressed as $\mathcal{X}_{i j k}$.

Fibers are vectors generated by fixing all but one index constant. $\mathcal{X}_{:, j, k}, \mathcal{X}_{i,:, k}, \mathcal{X}_{i, j,:}$ are the examples of fibers. Similarly, slices are generated by fixing all but two indices constant, e.g., $\mathcal{X}_{:,:, k}$,

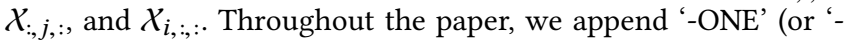
ALL') to the names of current state-of-the-art tensor factorization frameworks/implementations that maintain one (or $d$ ) CSF representation(s) of the input tensor. Table 1 summarizes the rest of the terminologies used throughout the paper. 


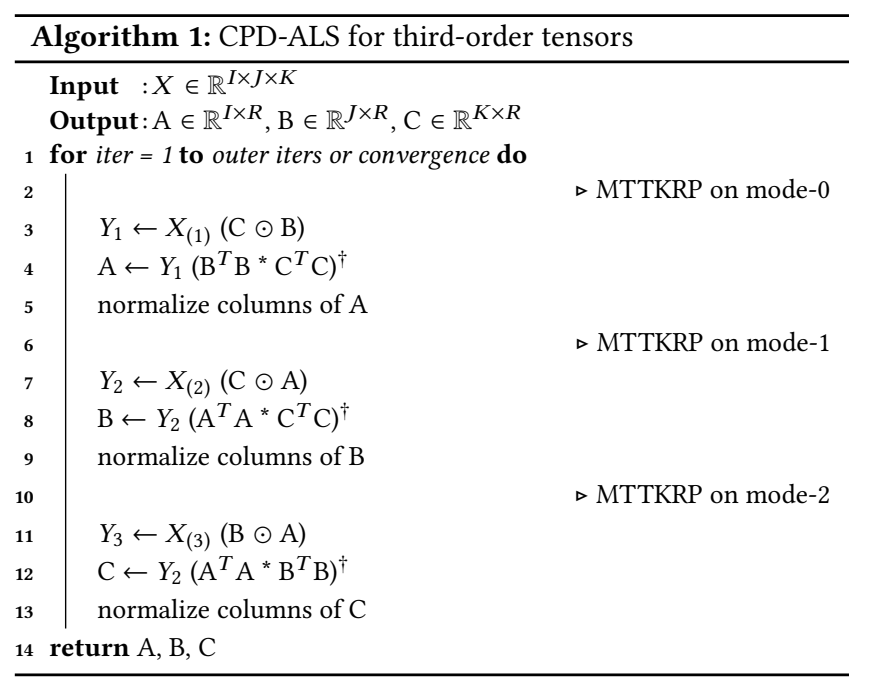

\subsection{CANDECOMP/PARAFAC Decomposition and MTTKRP}

CPD is the higher level generalization of Singular Value Decomposition (SVD), a popular matrix decomposition technique. CPD decomposes a tensor into a sum of component rank-one tensors. For example, the third-order tensor $\mathcal{X}$ is decomposed to $a_{r} \in \mathbb{R}^{I}$, $b_{r} \in \mathbb{R}^{J}$ and $c_{r} \in \mathbb{R}^{K}$, where $a_{r}, b_{r}, b_{r}$ are the rank-one components of the factor matrices $A, B$ and $C$ respectively [21]. We can express the decomposition as:

$$
\mathcal{X} \approx \sum_{r=1}^{R} a_{r} \circ b_{r} \circ c_{r}
$$

We implement the alternating least squares (ALS) algorithm [11], [15] to perform CPD in this work, which is also known as the workhorse algorithm of it [21]. ALS aims to approximate $\mathcal{X}$ and minimize $\|X-\widetilde{X}\| . \widetilde{X}$ is the approximated tensor generated from the factor matrices (refer to Equation (1)). ALS solves one matrix at a time while holding the others constant. For example, while updating $A$, matrices $B$ and $C$ will be fixed. The update computation can be described as:

$$
A=X_{(1)}(C \odot B)\left(B^{T} B * C^{T} C\right)^{\dagger} .
$$

The term $X_{(1)}(C \odot B)$ represents Khatri-Rao product between $X_{(1)}$, $B$, and $C$; the algorithm computing it is known as the MTTKRP (Matricized Tensor Times Khatri-Rao Product). $X_{(1)}$ is the mode-1 matricization of $\mathcal{X}$. The output of the Khatri-Rao product is then multiplied with $\left(B^{T} B * C^{T} C\right)^{\dagger}$, which is the pseudo-inverse of the $R \times R$ matrix generated by $B^{T} B$ and $C^{T} C$. Algorithm 1 demonstrates the steps to update each matrix using the ALS algorithm. Line 3, Line 7 , and Line 11 show the MTTKRP operations to update $A, B$, and $C$ respectively. MTTKRP is the performance bottleneck in CPD, primarily due to the access to the large, sparse tensor $\mathcal{X}$, and the scattered access to the factor matrices directed from $X$.

\subsection{The Optimized MTTKRP Algorithm}

In the MTTKRP computation, if $(C \odot B)$ is computed separately, then the resulting dense $J K \times R$ matrix could cause memory overflow.

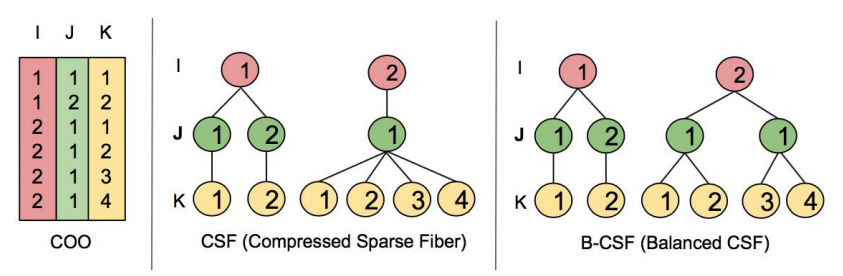

Figure 2: Storage formats of an illustrative sparse tensor

One way to avoid this issue is proposed by Kang et al. [19], which uses operator distributivity to compute the Hadamard products between $B$ and $X$, and $C$ and $X$. Based on this algorithm, Smith et al. [31] provide an optimized formulation to save flops and memory accesses, as shown below:

$$
\begin{aligned}
Y_{1}(i,:) & =\sum_{k=0}^{K-1} \sum_{j=0}^{J-1} X(i, j, k)(B(j,:) * C(k,:)) \\
& =\sum_{k=0}^{K-1} C(k,:) * \sum_{j=0}^{J-1} X(i, j, k) *(B(j,:))
\end{aligned}
$$

In Equation (4), each $\mathcal{X}_{(i,:, k)}$ fiber has a scope to save $R(J-1)$ multiplications.

\section{SPARSE TENSOR FORMATS}

There are two primary families of sparse tensor formats: coordinatebased and tree-based. A straightforward approach to represent a sparse tensor is to store, for each nonzero, its indices along each dimension and the value. This storage format is called COO (Coordinate) format. Recently, Li et al. [23] proposed Hierarchical COO (HiCOO) format based on $\mathrm{COO}$, which further compresses indices by multi-level blocking. Flagged-COO format [25] also belongs to the coordinate family. The tree-based format family compresses the sparse tensor indices into a "tree structure". CSF, proposed by Smith et al. [35], and BCSF, proposed by Nisa et al. [26], belong to this family. Since the tree-based formats are the focus of this work, we now analyze CSF and BCSF formats in terms of storage, floating point operations, memory access, and the number of required representations.

\subsection{Storage and Floating Point Operations}

Figure 2 shows the data structures of COO, CSF, and BCSF for a third-order tensor. $\mathrm{COO}$ requires $3 \times 4 \times M$ bytes to store the indices, where $M$ is the total number of nonzeros, and each index is a 4-byte integer. CSF organizes the dimension of the tensor in a hierarchical manner and compresses repetitive indices. As shown in the CSF tree in Figure 2(b), the leaves at the lowest level store the indices of the $M$ nonzeros at $K$ dimension. The nonzeros sharing the same indices at $J$ dimension are compressed to $F$ fibers, and the fibers sharing the same indices at $I$ dimension are grouped into $S$ slices/roots. This particular hierarchical organization can be represented as $I \rightarrow J \rightarrow K$. In general, such hierarchical organizations are called mode orientation. Thus, a tensor with $S$ slices, $F$ fibers, and $M$ nonzeros requires $4 \times(2 S+2 F+M)$ bytes to represent its indices. For slices and fibers, two arrays are maintained to store 

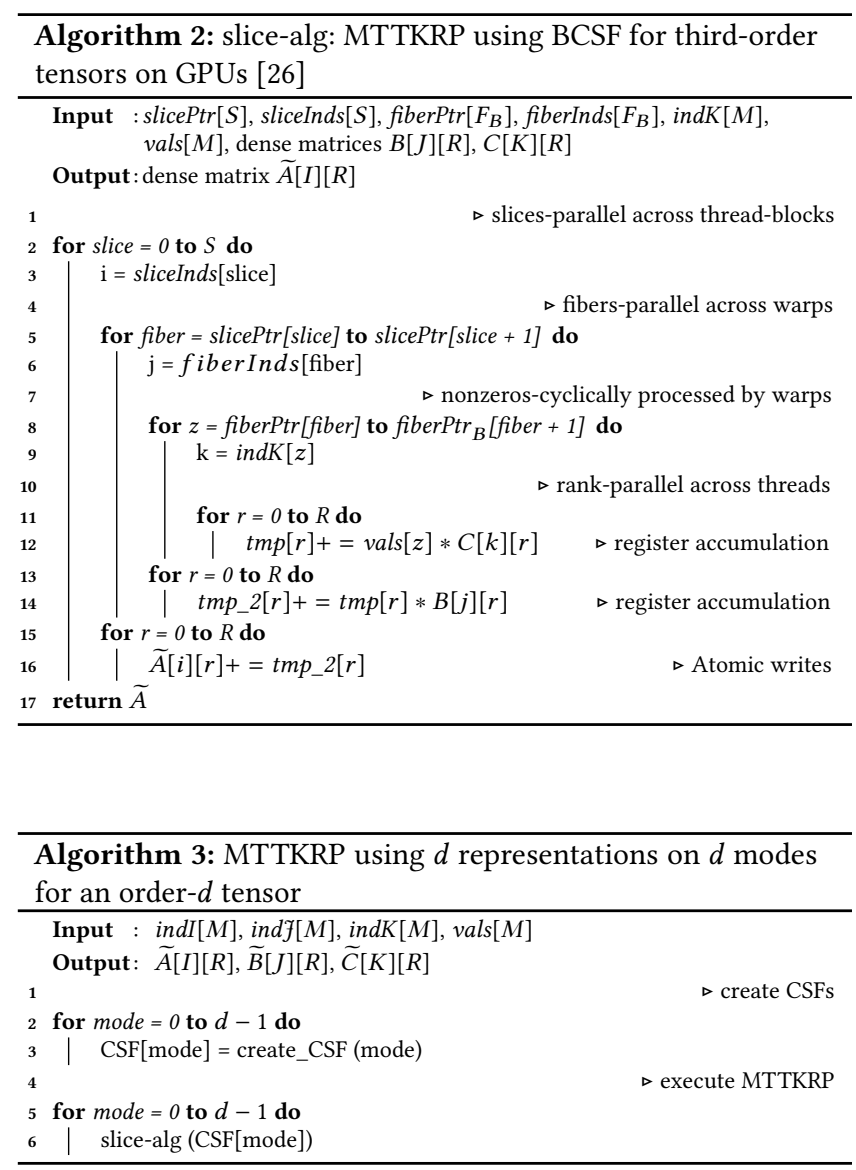

their pointers and indices. SPLATT [35] library provides a highly optimized MTTKRP implementation using CSF data structures on the CPU. Figure 2(c) shows the balanced CSF structure. BCSF [26] extends CSF to GPU platforms, and provides a balanced data structure to store the CSF. The nonzero elements per fiber and per slice might vary significantly across a tensor. BCSF splits the heavy fibers into sub-fibers and creates $F_{B}$ fibers, where $F_{B} \geq F$. The slices are grouped into multiple bins based on their length; each bin will have a different number of thread blocks assigned to one slice. BCSF increases GPU occupancy significantly with load balancing and other relevant optimizations [26]. Algorithm 2 shows the computation of MTTKRP on mode- 0 to update $A$ using BCSF. The first for loop as shown in Line 2 will iterate over all the slices; each slice will in turn iterate over its fibers in Line 2. Each nonzero element of the fibers will perform a multiplication with the corresponding row of $C$. The product will then be multiplied with the corresponding row of $B$ (Line 10) and written back to the row of $A$ (Line 16).

In real-world tensors, $S \ll M$ and $F \ll M$. Hence, CSF-based structures have a potential to reduce the floating point operations as well. As shown in Equation (4), and also in Algorithm 2, $R(J-1)$ flops can be saved by factoring $C$ out; doing so reduces the required flops to $R(S+2 \times(F+M))$. In contrast, the flops requirement of COO-based formats is $3 \times M \times R$.

\begin{tabular}{c|cccc|cccc}
\hline & \multicolumn{9}{|c|}{ fiber length of the selected mode } & \multicolumn{4}{c}{ fiber length of a candidate mode } \\
\hline & $0 \sim 100$ & $100 \sim 5 \mathrm{~K}$ & $5 \mathrm{~K} \sim 10 \mathrm{~K}$ & $>10 \mathrm{~K}$ & $0 \sim 100$ & $100 \sim 5 \mathrm{~K}$ & $5 \mathrm{~K} \sim 10 \mathrm{~K}$ & $>10 \mathrm{~K}$ \\
\hline deli & $37 \mathrm{M}$ & $76 \mathrm{~K}$ & 17 & 6 & $47 \mathrm{M}$ & $3 \mathrm{~K}$ & 5 & 0 \\
nell1 & $17 \mathrm{M}$ & $136 \mathrm{~K}$ & 233 & 77 & $113 \mathrm{M}$ & $11 \mathrm{~K}$ & 4 & 0 \\
nell2 & $46 \mathrm{~K}$ & $291 \mathrm{~K}$ & 5 & 0 & $16 \mathrm{M}$ & $66 \mathrm{~K}$ & 0 & 0 \\
flick & $13 \mathrm{M}$ & $166 \mathrm{~K}$ & 70 & 11 & $28 \mathrm{M}$ & $1 \mathrm{~K}$ & 1 & 0 \\
fr_m & $\mathbf{6 1 M}$ & $\mathbf{1 9 . 4 K}$ & $\mathbf{4 2}$ & $\mathbf{3 2}$ & $\mathbf{6 0 M}$ & $\mathbf{1 9 . 6 K}$ & $\mathbf{4 8}$ & $\mathbf{5 0}$ \\
fr_s & $\mathbf{9 1 M}$ & $\mathbf{2 8 K}$ & $\mathbf{9 0}$ & $\mathbf{5 9}$ & $\mathbf{8 6 M}$ & $\mathbf{3 0 K}$ & $\mathbf{1 1 9}$ & $\mathbf{1 0 5}$ \\
darpa & $54 \mathrm{~K}$ & $22 \mathrm{~K}$ & 164 & 277 & $28 \mathrm{M}$ & 0 & 0 & 0 \\
\hline
\end{tabular}

Table 2: Comparison of fiber lengths (nonzeros per fiber) between the selected mode by SPLATT-ONE and a candidate/non-selected mode

\subsection{Number of Representations}

CSF based representations are compressed with respect to a certain mode. Therefore, one may need to maintain $d$ distinct representations to exploit the compression at each mode. For example, the third-order tensor shown in Figure 3(a) would require three CSF representations: CSF-0, CSF-1, and CSF-2; they are shown in Figure 3(b). Algorithm 2 (slice-alg) shows the computation of MTTKRP on mode- 0 to update $A$ using CSF- 0 , where $i$ indices are at the slice mode. The intermediate modes in this case can either be mode- 1 or mode-2. Similarly, to perform MTTKRP on mode- 1 to update $B$, CSF-1 will be used and the $j$ indices will be at the slice mode. A similar analogy can be drawn for mode-2. Both SPLATT and BCSF by default use $d$ representations for an order- $d$ tensor. In both the frameworks, the mode orientation are such that, at mode- $n$, dimension $n$ will be at the root, and the rest of the modes will be sorted according to their dimension length to achieve the most compressed representation. Algorithm 3 shows the steps to perform MTTKRP at $d$ modes. At Line $3, d$ number of CSFs are created, and at Line 6, MTTKRP operations are performed at each mode.

$\mathrm{COO}$ on the other hand is invariable to mode orientation, and uses a single representation to compute MTTKRP at all $d$ modes. SPLATT library also supports SPLATT-ONE, i.e., using a single representation to perform MTTKRP on $d$ modes [31]. For example, since the tensor in Figure 3(a) is of dimension $3 \times 5 \times 6$, CSF- 0 will be selected as the SPLATT-ONE representation - the shortest mode, $I$, is slice mode, $J$ is the fiber mode, and the longest mode, $K$, is the nonzero mode. Although in this case, MTTKRP computation at only one mode (e.g., mode- 0 in CSF- 0 ) will benefit from the compression at both fiber- and slice-level. At the other modes (e.g., mode- 1 and mode-2 in CSF-0), MTTKRP will be computed using fiber-alg (Algorithm 7) exploiting compression only at the fiberlevel, and using nonzero-alg at the nonzero-level (Algorithm 8) with no possible compression. We describe these algorithms in details in the next section. Algorithm 4 shows MTTKRP computation on $d$ modes using a single representation. At Line 1 , the dimension for the slice-, fiber-, and nonzero-level is chosen according to the length of the dimensions. MTTKRP on mode- $0 /$ mode $-1 /$ mode- 2 is performed at Line 6/Line 8/Line 10. Using the 2D matrix analogy, computing MTTKRP at mode 1 using CSF- 0 is similar to performing SpMTV using a CSR representation instead of CSC.

\section{MM-CSF: A MIXED-MODE CSF}

SPLATT-ALL and BCSF-ALL iteratively update the matrices which correspond to the mode at its root/slice level. For a third-order 


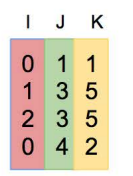

(a) Tensor $X(3 \times 5 \times 6)$

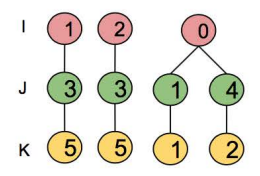

CSF-0

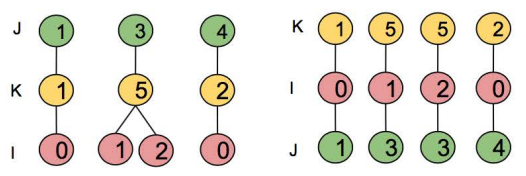

CSF-1
CSF-2

(b) SPLATT-ALL (CSF with $d$ representations)

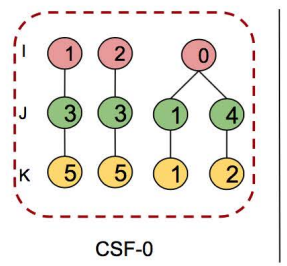

(c) Selected as SPLATT-ONE

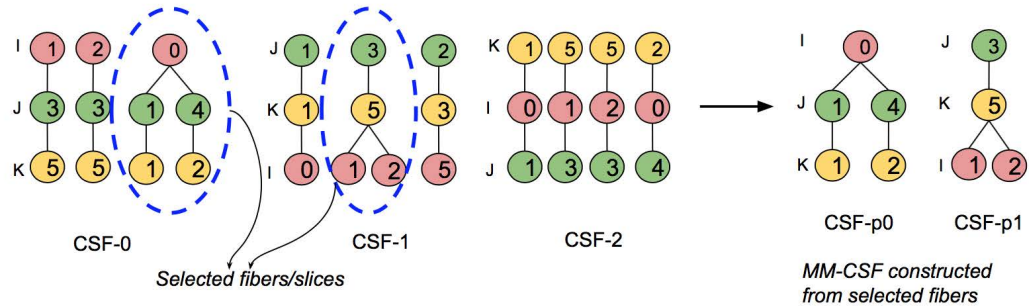

(d) Construction of MM-CSF (Mixed-Mode CSF)

Figure 3: Construction of MM-CSF

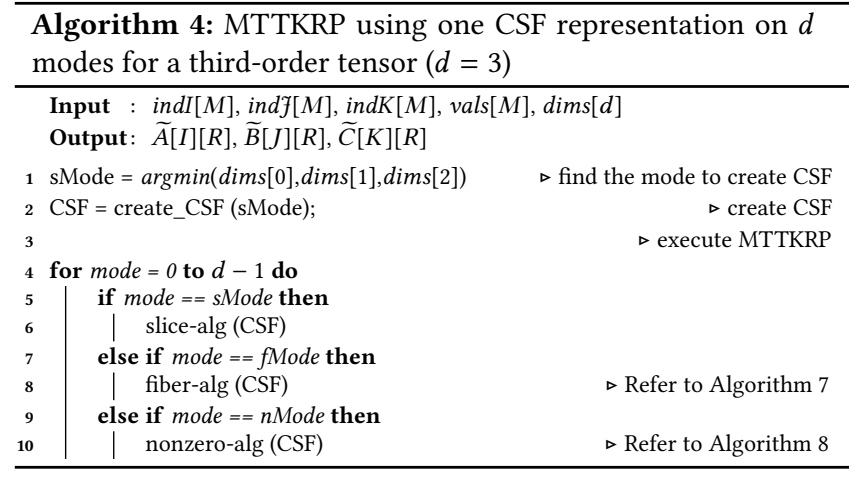

tensor $\mathcal{X}$, matrix $A, B$ and $C$ are updated sequentially using CSF-0, CSF-1, and CSF-2 respectively, when mode- 0 , mode-1, and mode- 2 are at the slice level. In the case of SPLATT-ONE representation, one of these three matrices will use the mode at slice-level to update itself, and the remaining two matrices will update the matrix at fiber-level and nonzero-level respectively. For SPLATT-ONE, the shortest dimension is selected as the slice (root) nodes, and the longest as the nonzero (leaf) nodes; the intermediate modes are sorted accordingly. The selected CSF thus has the highest average slice length, which leads to a high slice compression compared to other CSFs. However, the compression in the next fiber level is not always guaranteed. As shown in Table 2, for tensors fr_s and fr_m, instead of the CSF representation selected as SPLATT-ONE, other CSF representations can have higher average fiber length, and a higher number of fibers with length $>10 \mathrm{~K}$. This phenomenon can be more severe for higher order tensors. To incorporate multi-level compression, we propose MM-CSF, a Mixed-Mode CSF representation, where heavy fibers and slices are stored at their most suitable modes. For a 2D matrix, this would imply storing the dense rows in CSR format, and the dense columns in CSC format. While performing $y=A x$, the CSR part can efficiently parallelize across rows. Similarly, while performing $y=A^{T} x$, the CSC part can efficiently parallelize across columns.

\subsection{Partitioning of Nonzeros}

The first step in forming MM-CSF is to create $d$ disjoint partitions of the nonzero elements of an order- $d$ tensor $\mathcal{X}$, where each partition will have a different mode orientation. This aims to ensure that each nonzero goes to a partition where it has the potential to achieve the maximum compression. Thus, the resulting tensor partitions should jointly be more compressed, a.k.a. have fewer, but longer fibers. Figure 3(d) shows the construction of MM-CSF.

A nonzero element $X_{i, j, k}$ of a $3 \mathrm{D}$ tensor belongs to three ${ }^{2}$ possible fibers: fiber- 0 of CSF- 0 , fiber- 1 of CSF-1, and fiber- 2 of CSF-2. Let us assume that the lengths of fiber- 0 , fiber- 1 , and fiber- 2 are $l_{0}, l_{1}$, and $l_{2}$, respectively, and $l_{0}>l_{1}>l_{2}$. As fiber- 0 is the longest fiber, $\mathcal{X}_{i, j, k}$ will be assigned to the partition $P_{0}$ orientated in mode- 0 . When multiple fibers that the nonzero belongs to have same length, to break the tie, we assign the nonzero to the partition where average fiber length is higher. Partitions $P_{1}$ and $P_{2}$ will be similarly constructed.

In Figure 4, we demonstrate this strategy using fibers $\mathcal{X}_{:, j, k}$ and $\mathcal{X}_{i, j,:}$ in the columns under static strategy. All the nonzeros except $\mathcal{X}_{0,1,2}$ are assigned to partition $P_{0}$. However, this partitioning scheme creates an additional fiber by splitting fiber $\mathcal{X}_{0,1, \text { into two }}$ partitions. This shortens the length of fiber $\mathcal{X}_{0,1}$ : but increases the total number of fibers, leading to inefficient compression. The main reason is that the partitioning decision is based on statically pre-calculated fiber lengths at each mode orientation. We propose a dynamic strategy by incrementally updating the fiber length. Continuing with the aforementioned example, once the nonzero $X_{i, j, k}$ is assigned to partition $P_{0}$ since the length of fiber- $0\left(l_{0}\right)$ is the largest, the lengths of fiber-1 and fiber- 2 will be reduced by

\footnotetext{
${ }^{2}$ For ease of explanation, we use natural mode orientations, i.e., $\mathrm{I} \rightarrow \mathrm{J} \rightarrow \mathrm{K}$ for CSF- 0 , $\mathrm{J} \rightarrow \mathrm{K} \rightarrow \mathrm{I}$ for CSF- 1 , and $\mathrm{K} \rightarrow \mathrm{I} \rightarrow \mathrm{J}$ for CSF- 2 . In reality, $d$ ! mode orientations are possible, and the actual mode orientations of each CSF may differ from natural ones.
} 


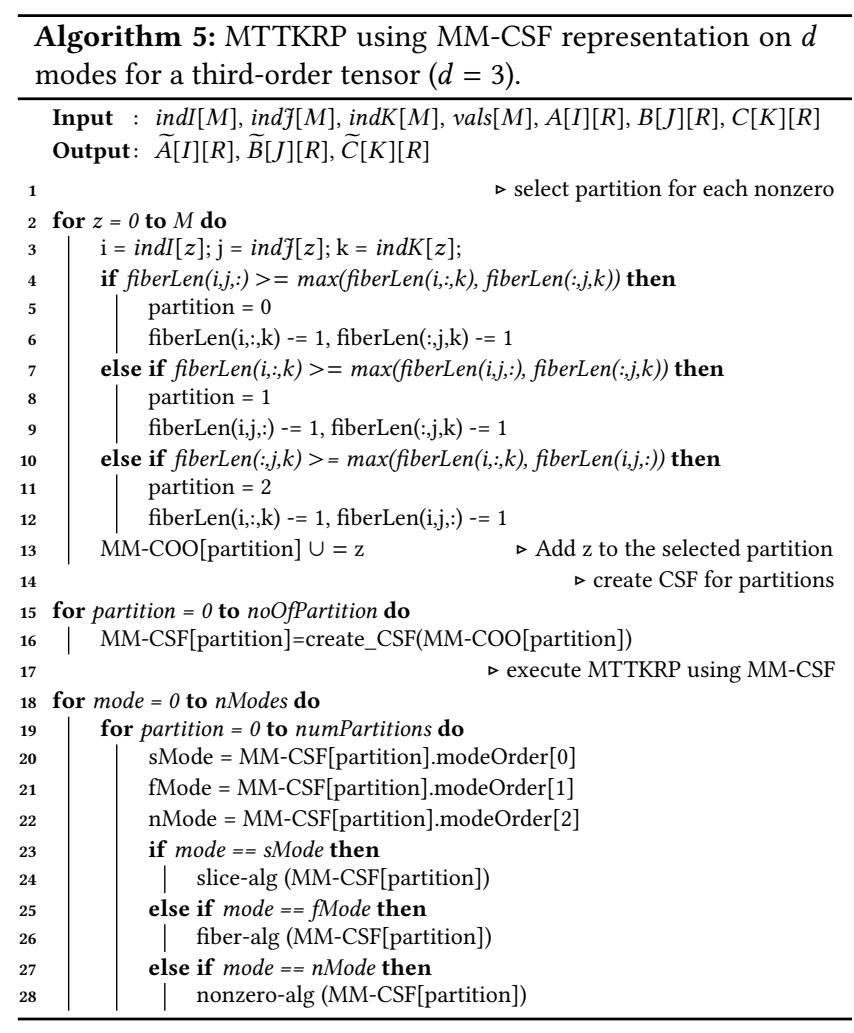

1 to record the processed nonzero. This is illustrated under the Figure 4 dynamic strategy columns. The pre-calculated length of fiber $\mathcal{X}_{1,2, \text { is }} 3$. Under static partitioning strategy, nonzero $\mathcal{X}_{0,1,2}$ will be assigned to partition 1 , which creates a fiber in partition 1 with only one nonzero. This is not an efficient compression. On the

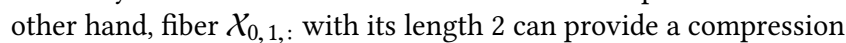
of 2 nonzeros. Compared to static partitioning, we have one less fiber and better compression after dynamic partitioning. Therefore, dynamic partitioning strategy reduces storage requirement, provides better compression, and consequently improves performance.

Algorithm 5 demonstrates the construction of MM-CSF partitions. The construction scheme and the algorithms shown in this paper are for $3 \mathrm{D}$ tensors. The extension to arbitrary dimensions is straightforward. The for loop (Line 2) determines the partitions of all nonzeros. A nonzero is assigned to the one with the longest fiber length among fiber-0, fiber- 1 and fiber-2, shown in Line 4 to 13 After all the nonzeros have been processed, MM-CSF is constructed by creating one CSF representation for each partition, like CSF- $p_{0}$, $\mathrm{CSF}-p_{1}$ in Figure $3(\mathrm{~d})$. To perform MTTKRP on a particular mode, each partition needs to perform its role by using either slice-, fiber-, or nonzero-centric algorithms. For example, to perform MTTKRP on mode- 0 , CSF- $p_{0}$ will use the (optimized) slice-alg (Algorithm 6), $\mathrm{CSF}-p_{1}$ will use the fiber-alg (Algorithm 7) and finally, CSF- $p_{2}$ will use the nonzero-alg (Algorithm 8). These algorithms will be described in Section 5.

\begin{tabular}{|c|c|c|c|c|c|c|c|c|c|c|}
\hline \multirow{3}{*}{$\begin{array}{c}\text { nonzero } \\
\mathrm{i}, \mathrm{j}, \mathrm{k}\end{array}$} & \multicolumn{6}{|c|}{ static strategy } & \multicolumn{4}{|c|}{ dynamic strategy } \\
\hline & \multicolumn{2}{|c|}{ fibers $<i, j>$} & \multicolumn{2}{|c|}{ fibers $<\mathrm{j}, \mathrm{k}>$} & \multirow{2}{*}{$\begin{array}{l}\text { Assig. } \\
\text { parti. }\end{array}$} & \multirow{2}{*}{$\begin{array}{l}\text { tot } \\
\mathrm{fbr}\end{array}$} & $\begin{array}{c}\text { fibers } \\
\langle\text { i.j.j> }\end{array}$ & $\begin{array}{l}\text { fibers } \\
<j, k>\end{array}$ & Assig. & tot \\
\hline & pair & len & pair & len & & & len & len & & \\
\hline 511 & $<5,1>$ & 3 & $<1,1>$ & 2 & 0 & 1 & 3 & 2 & 0 & 1 \\
\hline 512 & $<5,1>$ & 3 & $\langle 1,2\rangle$ & 3 & 0 & 1 & 3 & 3 & 0 & 1 \\
\hline 513 & $<5,1>$ & 3 & $\langle 1,3\rangle$ & 1 & 0 & 1 & 3 & 1 & 0 & 1 \\
\hline 412 & $<4,1>$ & 3 & $<1,2>$ & 3 & 0 & 2 & 3 & 2 & 0 & 2 \\
\hline 411 & $<4,1>$ & 3 & $<1,1>$ & 2 & 0 & 2 & 3 & 1 & 0 & 2 \\
\hline 415 & $<4,1>$ & 3 & $\langle 1,5\rangle$ & 1 & 0 & 2 & 3 & 1 & 0 & 2 \\
\hline 012 & $<0,1>$ & 2 & $\langle 1,2\rangle$ & 3 & 1 & 3 & 2 & 1 & 0 & 3 \\
\hline 014 & $<0,1>$ & 2 & $<1,4>$ & 1 & 0 & $(4)$ & 2 & 1 & 0 & \\
\hline
\end{tabular}

Figure 4: Adjusting fiber lengths during partitioning

\begin{tabular}{c|c|c|c|c}
\hline \multicolumn{1}{c|}{ Formats } & FLOPS & $\begin{array}{c}\text { Reads on } \\
\text { matrices }\end{array}$ & $\begin{array}{c}\text { Writes on } \\
\text { matrices }\end{array}$ & $\begin{array}{c}\text { Storage of } \mathcal{X} \\
\text { in words }\end{array}$ \\
\hline COO & $3^{*} 3 \mathrm{MR}$ & $3^{*} 2 \mathrm{MR}$ & $3^{*} \mathrm{MR}$ & $3^{*} \mathrm{M}$ \\
SPLATT-ALL & $3^{*}(\mathrm{~S}+2 \mathrm{~F}+2 \mathrm{M}) \mathrm{R}$ & $3^{*}(\mathrm{~F}+\mathrm{M}) \mathrm{R}$ & $3^{*} \mathrm{SR}$ & $3^{*} 2(\mathrm{~S}+\mathrm{F})+\mathrm{M}$ \\
SPLATT-ONE & $(\mathrm{S}+4 \mathrm{~F}+5 \mathrm{M}) \mathrm{R}^{1}$ & $3^{*}(\mathrm{~F}+\mathrm{M}) \mathrm{R}$ & $(\mathrm{S}+\mathrm{F}+\mathrm{M}) \mathrm{R}^{2}$ & $2(\mathrm{~S}+\mathrm{F})+\mathrm{M}$ \\
\hline \multirow{3}{*}{ MM-CSF } & $\left(\mathrm{S}_{P 0}+4 \mathrm{~F}_{P 0}+5 \mathrm{M}_{P 0}\right) \mathrm{R}$ & $\left(\mathrm{F}_{P 0}+\mathrm{M}_{P 0}\right) \mathrm{R}$ & $\left(2 \mathrm{~F}_{P 0}+\mathrm{M}_{P 0}\right) \mathrm{R}$ & $3\left(\mathrm{~F}_{P 0}+\mathrm{F}_{P 1}+\mathrm{F}_{P 2}\right)$ \\
& $+\left(\mathrm{S}_{P 1}+4 \mathrm{~F}_{P 1}+5 \mathrm{M}_{P 1}\right) \mathrm{R}^{3}$ & $+\left(\mathrm{F}_{P 1}+\mathrm{M}_{P 1}\right) \mathrm{R}$ & $+\left(2 \mathrm{~F}_{P 1}+\mathrm{M}_{P 1}\right) \mathrm{R}$ & $+\mathrm{M}_{P 0}+\mathrm{M}_{P 1}+\mathrm{M}_{P 2}$ \\
\hline & $+\left(\mathrm{S}_{P 2}+4 \mathrm{~F}_{P 2}+5 \mathrm{M}_{P 2}\right) \mathrm{R}$ & $+\left(\mathrm{F}_{P 2}+\mathrm{M}_{P 2}\right) \mathrm{R}$ & $+\left(2 \mathrm{~F}_{P 2}+\mathrm{M}_{P 2}\right) \mathrm{R}$ & $+{ }^{2}$
\end{tabular}

${ }^{1}(\mathrm{~S}+4 \mathrm{~F}+5 \mathrm{M}) \mathrm{R}=$ slice-mode: $(\mathrm{S}+2 \mathrm{~F}+2 \mathrm{M}) \mathrm{R}$, fiber-mode: $2(\mathrm{~F}+\mathrm{M}) \mathrm{R}$, nonzero-mode: $3 \mathrm{MR}$

${ }^{2}(\mathrm{~S}+\mathrm{F}+\mathrm{M}) \mathrm{R}=$ slice-mode: $\mathrm{SR}$, fiber-mode: $\mathrm{FR}$, nonzero-mode: $3 \mathrm{MR}$

${ }^{3} S_{P x}: S$ in partition $x, F_{P x}: F$ in partition $x, M_{P x}: M$ in partition $\mathrm{x}$

Table 3: Theoretical comparison between formats in terms of storage, flop computation, read and write transactions.

\section{BALANCED MTTKRP ALGORITHMS USING MM-CSF}

The current state-of-the-art, BCSF-ALL [26], only performs slice-alg for MTTKRP on GPUs. We propose balanced fiber-alg and nonzeroalg for GPUs, and furthermore optimize the slice-alg of BCSF-ALL. BCSF-ALL can take advantage of our optimized slice-alg. However, the data structure to support these new algorithms consumes $(3 F+$ $M)$ space rather than $(2 S+2 F+M)$ in other formats; usually $F \gg S$. For MM-CSF, the number of fibers, $F$, can be significantly reduced (e.g., a $2 \times$ reduction in tensors $\mathrm{fr}_{-} \mathrm{s}$ and $\mathrm{fr} \_\mathrm{m}$ ) by applying the partitioning scheme described in Section 4 . Reduced fiber count not only improves space efficiency, but also improves the performance, as less fibers lead to reduced memory accesses. Table 3 shows a theoretical comparison of the read transactions, write transactions, floating-point operations (flop) etc., among COO, SPLATT-ALL, SPLATT-ONE and MM-CSF.

\subsection{MTTKRP on Slice mode}

Details of BCSF-ALL. Updating the matrix corresponding to the slice-level incurs a minimum number of global write operations, since the number of slices is traditionally less than the number of fibers or non-zeros for tensors. The BCSF-ALL scheme shown in Algorithm 2 comprises two steps. In the first step, all the nonzeros are reduced to the corresponding fiber (Line 14). The second step involves a subsequent reduction across all the fibers to the parent slice (Line 12). These two steps are collectively termed as slice-mode operation. The reductions are performed in registers since they have the lowest access latency in the GPU memory hierarchy. However, the indices of the nonzeros, the fibers and the slices must be read from the global memory, so that corresponding rows can be fetched from the factor matrices (Lines 6,8).

We use an illustrative example to demonstrate the total read/write computations involved in performing MTTKRP on mode- 0 


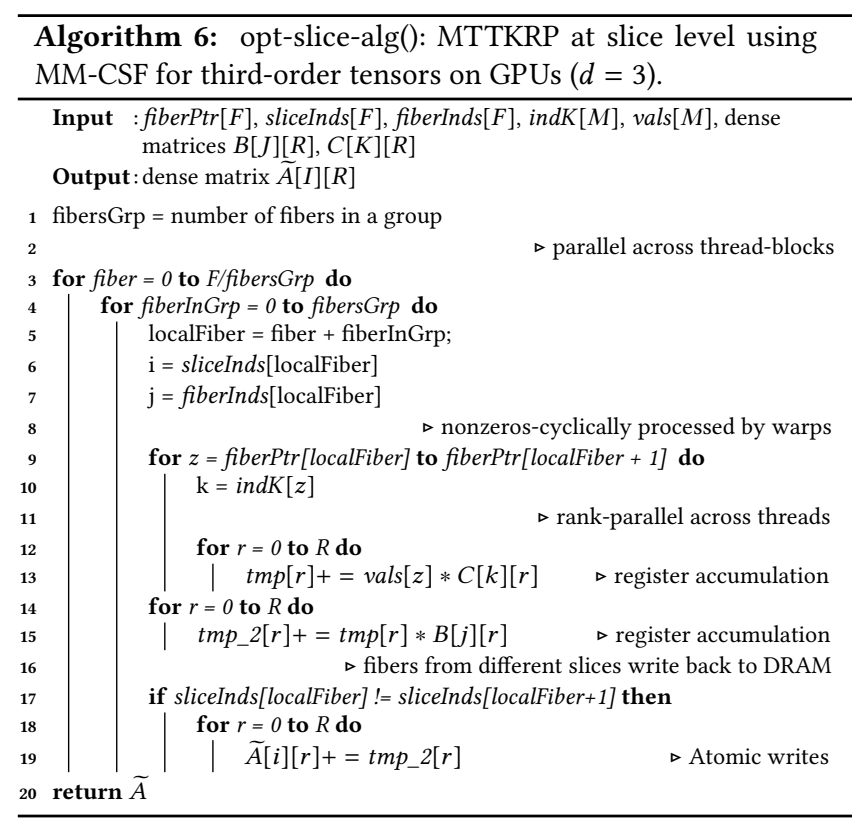

(i.e., MTTKRP at Line 2 of Algorithm 1) using the CSF-0 representation. Assume that the tensor $\mathcal{X}$ has only one slice, $\mathcal{X}_{i,: \text { :, }}$ and the slice has exactly two fibers, $\mathcal{X}_{i, x,:}$, and $\mathcal{X}_{i, y,:}$. Further, assume that each fiber has only three nonzeros, $\mathcal{X}_{i, x, p}, \mathcal{X}_{i, x, q}$ and $\mathcal{X}_{i, x, r}$ for fiber $\mathcal{X}_{i, x, \text {, }}$ and $\mathcal{X}_{i, y, p}, \mathcal{X}_{i, y, q}$ and $\mathcal{X}_{i, y, r}$ for fiber $\mathcal{X}_{i, y,:}$ In the slicemode scheme, each nonzero will read the rows with indices $p, q$, and $r$ from the dense matrix $C$, use these to perform the computation at Line 12 of Algorithm 2, and accumulate the result in registers $(t m p)$. Then, each fiber will read the rows with indices $x$ and $y$ from the dense matrix $B$, use these to perform the computation at Line 14 of Algorithm 2, and accumulate the result in registers ( $\left.t m p \_2\right)$. Finally, the slice will perform a read-modify-write to the row $i$ of $A$. In general, the total number of reads is $F$ (number of fibers) and $M$ (number of nonzeros); the number of read-modify-write is $S$ (number of slices) as shown in Table 3. The flop count varies from $2 M R$ to $5 M R$.

Improvement over BCSF-ALL. BCSF-ALL splits the exceptionally large slices into sub-slices, and assigns multiple thread-blocks to process each slice. In a similar spirit, multiple smaller slices can be assigned to the same thread-block. To select these assignments, extra pre-processing time and a separate data structure are maintained. The warps within a thread-block process the fibers (sub-fiber) in the respective slice. Each warp reduces the nonzeros of its fiber, and stores the accumulated result in a register. Figure 5(a) pictorially represents the slice-mode algorithm using BCSF-ALL. The scope of parallelism can be increased significantly by offering a finer-grained parallelism. In the proposed format (Figure 5(b)), we assign threadblocks to fibers instead of slices and warps to nonzeros instead of fibers. One limitation of this scheme is the increased number of global writes. Previously, fibers from the same slice would have accumulated the results in the register, and write back to global memory at the end. To incorporate this compression benefit, we group fibers into smaller chunks and assign one thread-block to

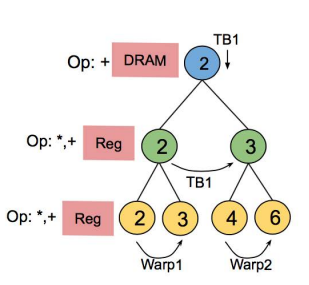

(a) slice-alg (Algorithm 2)

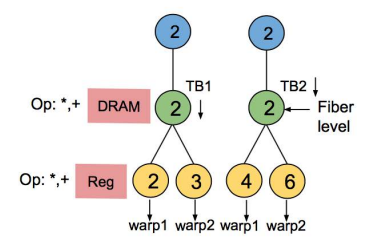

(c) fiber-alg

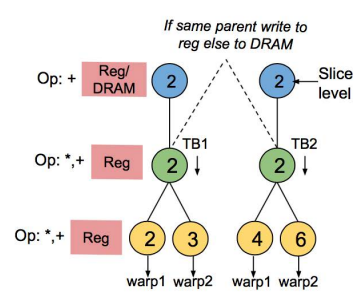

(b) opt-slice-alg (Algorithm 6)

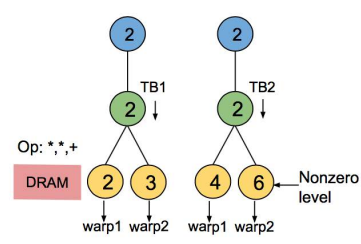

(d) nonzero-alg
Figure 5: MTTKRP algorithm variants

process a chunk. Fibers at each chunk check whether their parent slice is the same one or not. As long as they share the same slice, it keeps accumulating in the register, otherwise, writes back to global memory. Algorithm 6 demonstrates this optimized version of slice-alg. Line 3 iterates over the chunks and Line 4 iterates over the fibers at each chunk. Line 17 shows the comparison between the parent slices before writing back to global memory. Note that, this scheme might increase the number of atomic writes. Multiple chunks of fibers can share the same slice, and the number of atomics would increase with the number of chunks. To avoid race condition, atomic operations are used to accumulate the values from sub-slices of a slice.

In the original BCSF-ALL data structure, a fiber location was accessed via slice pointers. In matrix terminology, it would imply accessing the start point of the column indices using the row pointer. If we parallelize across the fibers, we need to directly access the fiber indices without fetching the start location from the slice pointers. One expensive way to achieve this is to perform a search to find parent slice of the fiber. Instead, we maintain an array of size $F$ to store the corresponding slice indices in lieu of two arrays (pointers and indices) of size $S$. Now, each thread-block can directly access the slice and fiber indices. This scheme outperforms BCSF-ALL by increasing parallelism while preserving the compression, as we demonstrate through evaluation in Section 6.

\subsection{MTTKRP on Fiber Mode}

We now describe the algorithm to compute MTTKRP on mode-1 (i.e., MTTKRP at Line 3 of Algorithm 1) to update matrix $B$ using CSF-0 representation. Algorithm 7 demonstrates the steps of the algorithm. We continue with the illustrative example of Section 5.1. This time, instead of updating matrix $A$ via the slice indices, we will update $B$ via the fiber indices. The indices of interest for $B$ are $x$ and $y$. Recall that the fibers are $\mathcal{X}_{i, x}$, and $\mathcal{X}_{i, y,:}$; these fibers will read the row $i$ from $A$, and rows $p, q$, and $r$ from $C$. Therefore, the number of read-modify-writes (i.e., atomics) is $\mathrm{F}$ (number of fibers), instead of S (number of slices) in the previous slice-mode 


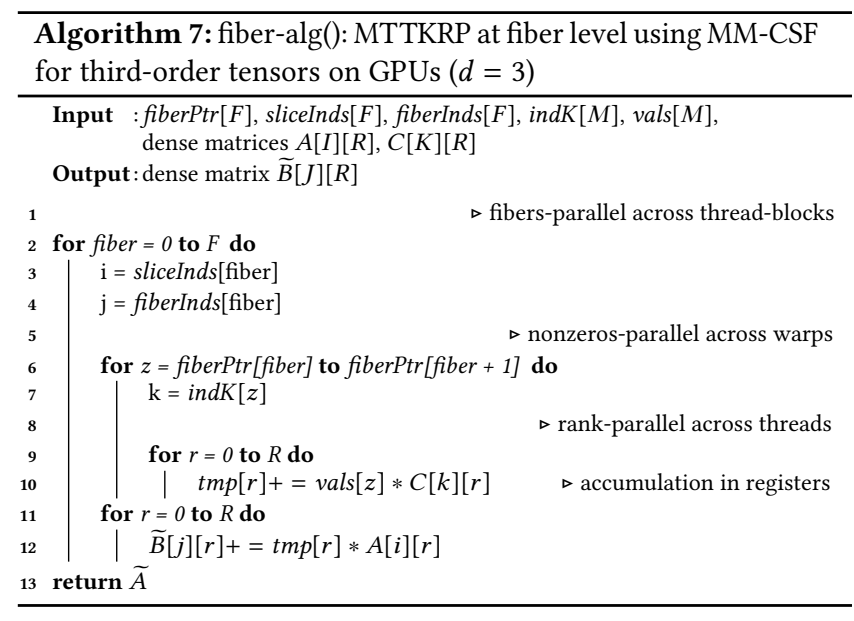

algorithm. The total number of reads decreases from $(F+M) R$ of the slice-mode algorithm to $(S+M) R$.

Some tensors inherently show good sparsity structure, for example, a low standard deviation in fiber length and slice length, clustered nonzeros in one mode, etc.; and a totally different structure in another mode, like power law structure. A mode offering a low number of writes with an imbalanced structure might underperform compared to a mode with a higher number of writes and better workload balance. A good example of such case is tensor Darpa. Darpa has 28 million nonzeros with the density of $2.37 E-9$. Both CSF- 0 and CSF- 1 have $22 \mathrm{~K}$ slices and $281 \mathrm{~K}$ fibers after splitting the long fibers. But the standard deviation of nonzero per slice is $60 \mathrm{~K}$ for mode 1 and $26 \mathrm{~K}$ for mode 0 . So, in terms of sparsity structure, CSF-0 is more balanced. If we use slice-mode on CSF-1 to compute MTTKRP on mode-1, the total number of reads and writes are $28.7 \mathrm{M}$ and $22 \mathrm{~K}$ respectively. Applying fiber-mode on CSF-0 will result in $28.5 \mathrm{M}$ reads and $281 \mathrm{~K}$ writes. Interestingly, $70 \%$ improvement is achieved by using fiber-mode than slice-mode on mode 1. We verified our intuition by collecting metrics from NVPROF [1] profiler provided by NVIDIA. The metric achieved_occupancy, defined as the ratio of the average active warps per active cycle to the maximum number of warps supported on an SM, increase to $60 \%$ with fiber-mode from $40 \%$ for slice-mode on NVIDIA P100.

This algorithm exposes an opportunity for finer grained parallelism and reduction in memory latency by allowing similar parallelization strategy like the slice-mode algorithm. Figure 5(c) demonstrates the parallelization techniques. Here, warps can still use registers to reduce the nonzeros, but not to accumulate the sum from fibers. This is because the write locations are now the fiber indices, and fibers from other slices might write to the same location. Hence, we need to use atomic operations to guarantee correctness. But increased parallelism often mitigates the shortcoming of having high atomic operations and achieves comparable performance.

\subsection{MTTKRP on nonzero mode}

In the nonzero-mode algorithm presented in Algorithm 8, the write locations are fetched from the nonzero locations to update matrix $C$ Referring back to the illustrative example of Section 5.1, the three nonzeros are $\mathcal{X}_{i, x, p}, \mathcal{X}_{i, x, q}$ and $\mathcal{X}_{i, x, r}$ and the update locations are

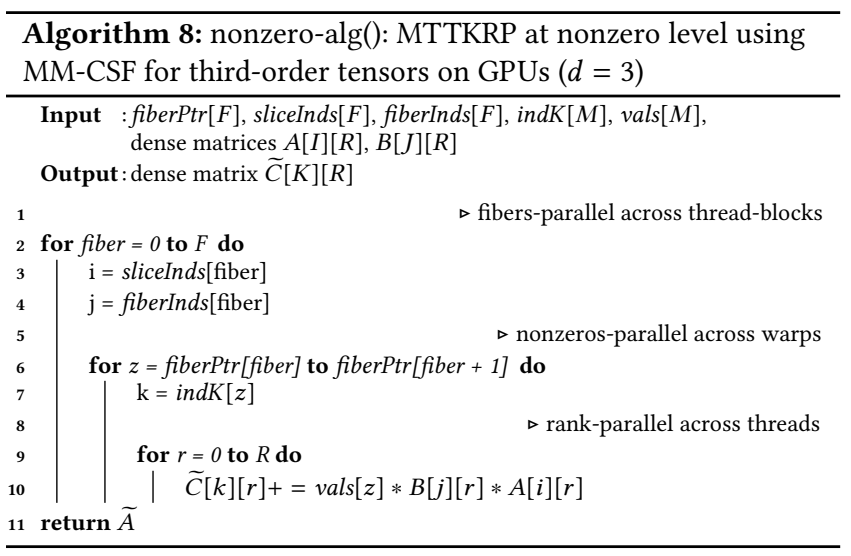

$p, q$ and $r$. Each nonzero reads row $i$ row of $A$ and, rows $x$ and $y$ of $B$. We adopt a similar parallelization strategy as fiber-mode. The total number of reads are further reduced to $(S+F) R$, and the number of writes increases to $M$. Figure $5(d)$ demonstrates the parallelization of this technique. Just like fiber-mode algorithm, the performance of this algorithm also depends on the sparsity structure of the tensor and the ratio between read and write. For example, Nell-1 dataset shows $11 \%$ improvement using nonzeromode algorithm on mode- 2 with CSF- 0 . Nell-1 has $140 \mathrm{M}$ nonzeros with a density of $9.05 E-13$. CSF-0 representation for Nell-1 has $2 \mathrm{M}$ slices and $17 \mathrm{M}$ fibers, and CSF-2 has $25 \mathrm{M}$ slices and $113 \mathrm{M}$ fibers. If we use CSF-2 to compute mode- 2 using slice-mode, the number of reads and writes are $253 \mathrm{M}$ and $25 \mathrm{M}$ respectively. On the other hand, if we use CSF-0, the number of reads and writes are $157 \mathrm{M}$ and $140 \mathrm{M}$.

\section{EXPERIMENTAL EVALUATION}

\subsection{Evaluation Setup}

We evaluate the performance of MM-CSF ${ }^{3}$ in computing MTTKRP, the computational kernel of a popular CANDECOMP/PARAFAC decomposition (CPD), against five publicly available state-of-the-art frameworks: SPLATT ${ }^{4}[35], \mathrm{BCSF}^{5}[26], \mathrm{F}^{-C} \mathrm{COO}^{6}[25]$, and ParTI $^{7}$ [22] which provides HiCOO [23] and COO implementations. We used the latest updated code in the SPLATT git repository instead of the release version, as suggested by the authors. Of these frameworks, HiCOO and SPLATT are CPU-based implementations; ParTI-COO ${ }^{8}$, BCSF [26], and F-COO [25] are GPU-based frameworks. SPLATT, BCSF, and F-COO each create $d$ representations for an order- $d$ tensor by default. Additionally, SPLATT provides extensions to select the number of representations for a tensor [31]. Therefore, we present comparisons against both $d$ representations (SPLATT-ALL), and single representation (SPLATT-ONE) for SPLATT. Tiling is enabled for SPLATT while collecting the performance data. For a fair comparison, we modify the default

\footnotetext{
${ }^{3}$ https://github.com/isratnisa/MM-CSF

${ }^{4}$ https://github.com/ShadenSmith/splatt

${ }^{5}$ https://github.com/isratnisa/B-CSF

${ }^{6}$ https://github.com/kobeliu85/mttkrp-gpu

${ }^{7}$ https://github.com/hpcgarage/ParTI

${ }^{8}$ Since the COO CPU of ParTI is significantly outperformed by HiCOO, we only evaluate $\mathrm{HiCOO}$ for CPU. All references to ParTI-COO refer to the GPU implementation.
} 


\begin{tabular}{lllll} 
Tensors & order & Dimensions & \#Nonzeros & Density \\
\hline deli & 3 & $533 K \times 17 M \times 2 M$ & $140 \mathrm{M}$ & $6.14 \mathrm{E}-12$ \\
nell1 & 3 & $3 M \times 2 M \times 25 M$ & $144 \mathrm{M}$ & $9.05 \mathrm{E}-13$ \\
nell2 & 3 & $12 K \times 9 K \times 29 K$ & $77 \mathrm{M}$ & $9.05 \mathrm{E}-13$ \\
flick & 3 & $320 K \times 28 M \times 2 M$ & $113 \mathrm{M}$ & $7.80 \mathrm{E}-12$ \\
fr_m & 3 & $23 M \times 23 M \times 166$ & $99 \mathrm{M}$ & $1.10 \mathrm{E}-09$ \\
fr_s & 3 & $39 M \times 39 M \times 532$ & $140 \mathrm{M}$ & $1.73 \mathrm{E}-10$ \\
darpa & 3 & $22 K \times 22 K \times 23 M$ & $28 \mathrm{M}$ & $2.37 \mathrm{E}-09$ \\
\hline nips & 4 & $2 K \times 3 K \times 14 K \times 17$ & $3 \mathrm{M}$ & $3.85 \mathrm{E}-04$ \\
enron & 4 & $6 K \times 6 K \times 244 K \times 1 K$ & $5 \mathrm{M}$ & $1.83 \mathrm{E}-06$ \\
ch-cr & 4 & $6 K \times 24 \times 77 \times 32$ & $54 \mathrm{M}$ & $1.48 \mathrm{E}-01$ \\
flick & 4 & $320 K \times 28 M \times 2 M \times 731$ & $113 \mathrm{M}$ & $1.07 \mathrm{E}-14$ \\
uber & 4 & $183 \times 24 \times 1 K \times 2 K$ & $3 \mathrm{M}$ & $5.37 \mathrm{E}-10$ \\
\hline \multicolumn{5}{c}{ Table $4 \cdot$ Sparse tensor datasets }
\end{tabular}

Table 4: Sparse tensor datasets
BCSF-ALL implementation of [26] to support BCSF-ONE (i.e., use a single BCSF representation for all modes). We extend the fiber splitting and binning concept used in BCSF-ALL to implement well-optimized fiber- and nonzero-mode algorithms for BCSF-ONE. $\mathrm{HiCOO}$ and ParTI-COO use a single representation $\mathrm{HiCOO}$ and COO respectively.

The GPU data is collected on an NVIDIA Volta V100 GPU with 16GB memory. It has $80 \mathrm{SMs}$ and a $6144 \mathrm{~KB}$ L2 cache. The CPU data is collected on a Dell PowerEdge R740: a two-socket server with 40 -core Intel Xeon 6148 . It has $384 \mathrm{~GB}$ memory with $2.40 \mathrm{GHz}$ clock frequency. The CUDA code is compiled with NVCC-9.2, and the CPU code is compiled with GCC-7.3.0. The execution on CPU is parallelized over 40 threads. The results are collected using singleprecision data type and tensor rank, $R$, is set to 32 .

The benchmarks comprise 3D and 4D sparse tensors collected from real-world applications. Datasets like deli (delicious), nell1 and nell2 (Never Ending Language Learner knowledge), flick (Flickr) are from The Formidable Repository of Open Sparse Tensors and Tools, FROSTT [30]. Darpa, fr_m (freebase-music) and fr_s (freebasesampled) are from the dataset used in HaTen2 [18]. Table 4 lists the tensor order, dimensions, number of nonzeros (\#Nonzeros), and the density of these tensors.

\subsection{Reduction in Fibers Using MM-CSF}

Table 5 shows the reduction in fibers with MM-CSF for 3D tensors, compared to BCSF-ALL and BCSF-ONE representation. To provide better work balance on GPU, long fibers are split into sub-fibers, which increases the number of fibers when compared to SPLATTALL. This trend can be observed for some tensors in Table 5, e.g., a $5 \%$ increase in fiber count for nell-2. However, compared to BCSFALL, MM-CSF achieves an average of $80 \%$ reduction in the total fiber count. For most of the benchmarks, we observe a reduction in fiber count with MM-CSF compared to BCSF-ONE as well. For fr_m and fr_s dataset, a reduction of 55\% (61M to 27M) and 50\% ( $91 \mathrm{M}$ to $45 \mathrm{M}$ ) respectively in fiber count is observed. The primary reason behind such drastic reduction in fiber count for fr_m and fr_s is the presence of long fibers in mode- 2 and mode- 0 , as noted in Table 2 of Section 4.

\begin{tabular}{c|ccc|ccc}
\hline & \multicolumn{3}{|c|}{ \#Fibers (millions) } & \multicolumn{3}{c}{ Reduction \% } \\
\hline & BCSF- & BCSF- & MM- & BCSF- & BCSF- & MM- \\
& ALL & ONE & CSF & ALL & ONE & CSF \\
\hline deli & 122 & 38 & 26 & 0 & 69 & 78 \\
nell1 & 149 & 18 & 18 & 0 & 88 & 88 \\
nell2 & 18 & 1 & 1 & -5 & 96 & 95 \\
flick & 55 & 14 & 9 & -1 & 75 & 83 \\
fr_m & 183 & 62 & 28 & 0 & 66 & 85 \\
fr_s & 269 & 92 & 45 & 0 & 66 & 83 \\
darpa & 29 & 0.28 & 0.28 & -1 & 99 & 99 \\
\hline
\end{tabular}

Table 5: Reduction in number of fibers using MM-CSF compared to other GPU based CSF formats. Reduction (\%) is shown compared to SPLATT-ALL.

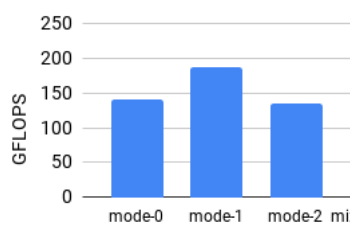

(a) fr_m

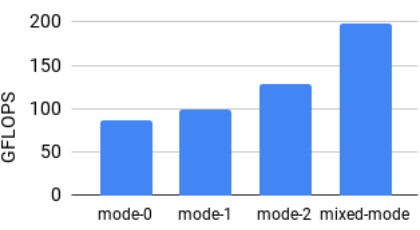

(b) fr_s
Figure 6: Achieved GFLOPS by assigning all elements to any single-mode vs. using a mixed-mode (MM).

\subsection{Impact of Partitioning}

We use the partitioning described in Section 4.1 for efficient compression. To evaluate the impact of the implemented partitioning scheme, we compare the results by assigning all elements of the tensor to a single partition against the mixed-mode partitioning, where nonzeros are assigned to multiple partitions. Both variations use the same underlying data structure for a fair comparison. Figure $6 \mathrm{a}$ and Figure $6 \mathrm{~b}$ demonstrate the benefit of partitioning on two representative tensors, fr_s, and fr_m. In both cases, the mixed mode partitioning creates two partitions and assigns nearly $50 \%$ nonzeros to each one. In both the tensors, we observe that performing mixed mode partitioning provides a significant performance improvement. On our evaluation over all $3 \mathrm{D}$ and $4 \mathrm{D}$ tensors, we consistently observe an improvement with partitioning over using an arbitrarily selected single representation.

\subsection{Improvement in GPU Occupancy and DRAM Transactions}

The kernels for MM-CSF increase GPU occupancy by applying finegrained parallelism. Table 6 documents the achieved occupancy for 3D tensors, measured via NVPROF. As evident, MM-CSF improves the device occupancy by $45 \%$ on average compared to BCSF-ALL. For fr_s dataset, the achieved occupancy improves by almost $2 \times$. Additionally, MM-CSF consistently reduces the global load transactions for all, and DRAM read transactions for majority of the tensors. In cases like deli, where the occupancy improvement is insignificant, the performance improvement can be attributed to a reduction in DRAM reads. However, MM-CSF incurs more DRAM 


\begin{tabular}{c|cc|cc|cc|cc}
\hline & \multicolumn{2}{|c|}{ GFLOPS } & \multicolumn{2}{c|}{ occup. in \% } & \multicolumn{2}{c|}{ glb. loads in GiB } & \multicolumn{2}{c}{ DRAM in GiB } \\
\hline & BCSF & MM- & BCSF & MM- & BCSF & MM- & BCSF & MM- \\
& -ALL & CSF & -ALL & CSF & -ALL & CSF & -ALL & CSF \\
\hline deli & 333 & 382 & 73 & 80 & 104 & 86 & 43 & 34 \\
nell1 & 270 & 285 & 68 & 77 & 112 & 80 & 55 & 55 \\
nell2 & 607 & 763 & 58 & 76 & 45 & 35 & 4 & 4 \\
flick & 327 & 435 & 50 & 79 & 76 & 59 & 33 & 27 \\
fr_m & 194 & 235 & 42 & 83 & 97 & 69 & 48 & 50 \\
fr_s & 203 & 228 & 53 & 84 & 140 & 102 & 70 & 73 \\
darpa & 209 & 327 & 35 & 52 & 28 & 13 & 12 & 11
\end{tabular}

Table 6: Improved occupancy, global loads, and DRAM read transactions using MM-CSF compared to BCSF-ALL (Data collected using NVPROF profiler on V100).

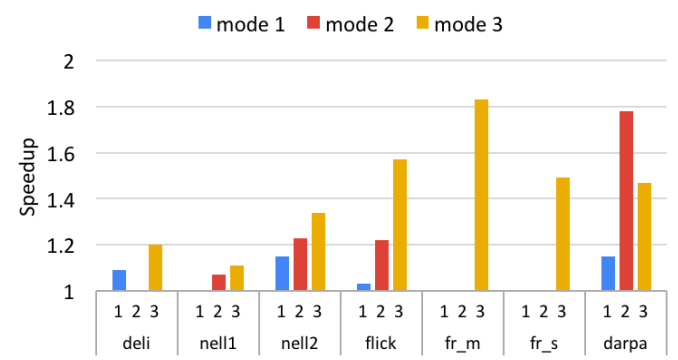

Figure 7: Speedup using MM-CSF compared to BCSF-ALL at $d$ modes on Tesla V100 GPU

transactions for fr_m and fr_s. This can be explained by the dimensionalities of these two tensors. For example, fr_m has dimensions $23 M \times 23 M \times 166$, which implies that $A$ and $B$ matrices are significantly large, $C$ matrix is small enough to be cached entirely in L2 cache of the Volta GPU. We compute the volume of data (in GiB) read from DRAM from the metrics collected by NVPROF. MM-CSF reads 17,16 and $16 \mathrm{GiB}$ data from DRAM in mode- 0 , mode- 1 and mode- 2 respectively, whereas BCSF-ALL reads 14,14 , and $20 \mathrm{GiB}$ data from DRAM. BCSF-ALL for fr_m has 60M fibers in each mode (refer to Table 5). Therefore, while updating $A$ and $B$ in mode- 0 and mode- 1 , the $60 \mathrm{M}$ accesses to the fibers come from $C$, which will likely to be cached in L2. However, while updating $C$ in mode-2, the fiber accesses come from $A$, resulting in a dramatic increase in DRAM reads. In contrast, with MM-CSF, all $A, B, C$ matrices will potentially be accessed at each mode due to the mixed-mode representation. This results in a consistent DRAM read in all modes, but slightly elevated DRAM reads in the first two modes compared to BCSF-ALL. However, the increase in DRAM transactions with MM-CSF in these two cases is compensated by an overall reduction in global memory transactions and the improved occupancy, resulting in performance enhancement over BCSF-ALL.

\subsection{Performance Comparison with BCSF-ALL}

An important metric to demonstrate the utility of MM-CSF is to show that by using it, one can match the performance achieved by the current state-of-the-art frameworks in computing MTTKRP, while simultaneously reducing the space requirement. To the best

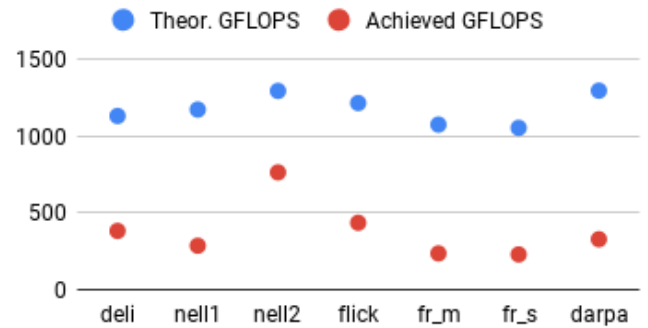

Figure 8: Achieved GFLOPS by MM-CSF compared to theoretically achievable GFLOPS in V100

\begin{tabular}{c|ccccccc}
\hline & $\begin{array}{c}\text { MM- } \\
\text { CSF }\end{array}$ & $\begin{array}{c}\text { BCSF- } \\
\text { ALL }\end{array}$ & $\begin{array}{c}\text { BCSF- } \\
\text { ONE }\end{array}$ & $\begin{array}{c}\text { PARTI } \\
\text { COO }\end{array}$ & $\begin{array}{c}\text { Hi- } \\
\text { COO }\end{array}$ & $\begin{array}{c}\text { SPLATT } \\
\text { ALL }\end{array}$ & $\begin{array}{c}\text { SPLATT } \\
\text { ONE }\end{array}$ \\
\hline deli & 106 & 121 & 125 & 149 & 5,403 & 5,342 & 3,284 \\
nell1 & 145 & 153 & 152 & 235 & 8,683 & 2,184 & 1,969 \\
nell2 & 29 & 37 & 37 & 71 & 262 & 140 & 94 \\
flick & 75 & 99 & 92 & 110 & 8,374 & 3,753 & 1,175 \\
fr_m & 122 & 148 & 208 & 225 & 5,136 & 5,021 & 6,897 \\
fr_s & 177 & 199 & 259 & - & 7,853 & 9,344 & 9,591 \\
darpa & 25 & 39 & 29 & 82 & 1,124 & 1,078 & 705 \\
uber & 3.72 & 2.6 & 4.05 & - & 298 & 93 & 109 \\
nips & 2.09 & 3.2 & 3.27 & - & 64 & 32 & 18 \\
chicago & 3.26 & 6.4 & 7.98 & - & 38 & 49 & 10 \\
flickr-4d & 130 & 176 & 183 & - & 5,632 & 9,392 & 2,076 \\
enron & 29 & 57 & 38 & - & 1,085 & 1,101 & 1,393 \\
\hline
\end{tabular}

Table 7: Time (ms) to run MTTKRP using MM-CSF and stateof-the-art benchmarks

of our knowledge, BCSF-ALL on GPU offers the maximum performance compared to the other existing frameworks. Figure 7 presents the speedup achieved by MM-CSF compared to BCSF-ALL for 3D tensors. On darpa and fr_m dataset, we outperform BCSFALL by a factor of $1.8 \times$. Consistent speedup is observed for the rest of the tensors. For the cases where BCSF-ALL already provides high occupancy, we do not observe any further speedup.

\subsection{Performance Model}

Figure 8 plots the achieved performance versus the theoretically achievable performance in computing MTTKRP on 3D tensors using MM-CSF representation. The theoretically achievable GFLOPS is computed by multiplying the operational intensity (OI) of MTTKRP kernel with the peak bandwidth of V100 GPU device. The gap between realized and theoretical peak performance of GPUs is challenging to bridge, even for compute-bound GEMM kernels. For MTTKRP, the significant gap can primarily be attributed to the poor data locality due to the sparsity of the input tensors. The performance gap is less pronounced for nell-2 dataset. This can be explained by the fact that it is the smallest among all evaluated tensors, with a dimension of $12 K \times 9 K \times 29 K$, and consequently has the highest $\mathrm{L} 2$ hit rate $(82 \%)$.

\subsection{Overall Performance}

Figure 9 shows the performance achieved by using MM-CSF as the representation to compute MTTKRP, against other state-of-theart representations/frameworks on both CPU and GPU platforms. 


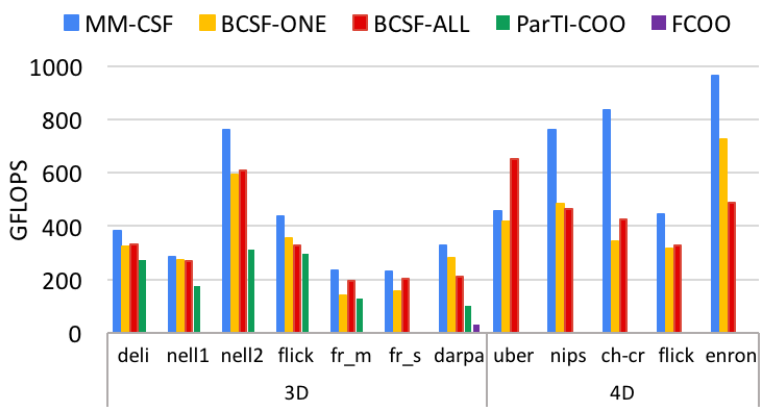

(a) GFLOPS comparison on an NVIDIA V100 GPU

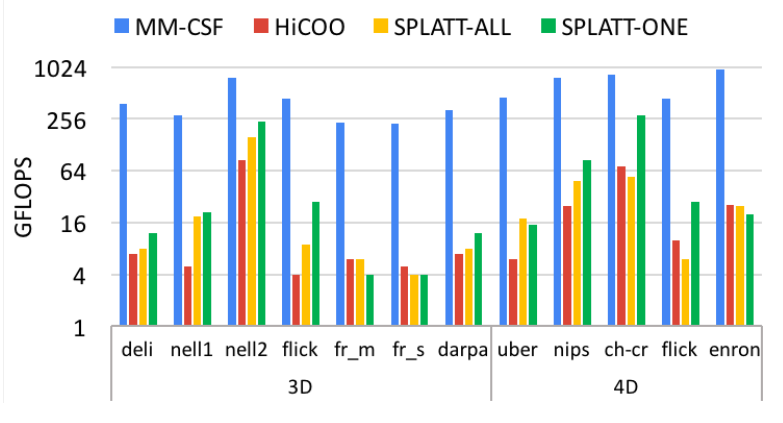

(b) GFLOPS comparison between MM-CSF (on GPU) and CPUbased framework on an Intel 40-core CPU.

Figure 9: Achieved performance of MM-CSF compared to other frameworks.

\begin{tabular}{cccccccc}
\hline & SPLATT & SPLATT & BCSF & ParTI & Hi & \multirow{2}{*}{ F-COO } & $\begin{array}{c}\text { MM- } \\
\text { CSF }\end{array}$ \\
\hline deli & -ALL & -ONE & -ALL & - COO & COO & & \\
nell1 & 3090 & 824 & 2691 & 1604 & 2955 & 3260 & 838 \\
nell2 & 1012 & 697 & 3010 & 1643 & 3062 & 3341 & 759 \\
flick & 1940 & 535 & 1019 & 880 & 250 & 1789 & 303 \\
fr_m & 2893 & 849 & 2894 & 1292 & 1309 & 2627 & 539 \\
fr_S & 4249 & 1232 & 4250 & 1601 & 1040 & 2316 & 700 \\
darpa & 723 & 109 & 726 & 325 & 200 & 3256 & 1051 \\
\hline
\end{tabular}

Table 8: Storage comparison in $\mathrm{MiB}$

For a uniform comparison, the floating-point operations of COOMTTKRP are used as a baseline in computing the GFLOPS for all the frameworks. MM-CSF achieves 510 GFLOPS on average, outperforming BCSF-ONE by a factor of $1.4 \times$, and ParTI-COO by a factor of $2 \times$ (Figure 9a). Note that the missing data for F-COO in Figure $9 \mathrm{a}$ is due to the failure of successful completion of MTTKRP computation at all modes. For nell2 dataset, MM-CSF achieves the highest performance of 966 GFLOPS.

Figure $9 \mathrm{~b}$ presents the performance comparison of MM-CSF with CPU-based formats. MM-CSF outperforms SPLATT-ALL by $35 \times$ on average. Recently published state-of-the-art COO-based format, $\mathrm{HiCOO}$, is $47 \times$ slower than MM-CSF. We also present the execution time of the CPU- and GPU-based benchmarks in Table 7.

\subsection{Overall Storage}

We present a comparison in space requirements of MM-CSF against state-of-the-art frameworks based on both CSR and COO format families in Table 8. We only use the indices to compute storage of the tensors, as storing the values of each nonzero needs the same space regardless of formats. MM-CSF significantly reduces the space requirement compared to SPLATT-ALL and BCSF-ALL. We now explain the slight increase observed in the storage requirement for MM-CSF compared to SPLATT-ONE. Apart from the fiber splitting for load balancing, MM-CSF also creates an extra data structure of size of $F$ to trace the slice indices along with the fiber indices. Despite these factors that can cause an increase in MM-CSF storage when compared to SPLATT-ONE, we observe an improvement in storage for fr_m and fr_s dataset with MM-CSF in Table 8.
GPU-based F-COO stores $d$ representations of the tensor in $\mathrm{COO}$ format. MM-CSF consumes $50 \%$ lower space than COO-based frameworks, and $40 \%$ lower space than HiCOO. A $3 \times$ space reduction is achieved for nell2 and darpa. This is expected as both of these tensors have long fibers and slices, providing good compression that only a CSF based format can exploit.

\subsection{Format Conversion to MM-CSF}

We compare the pre-processing time involved in constructing MMCSF vs. BCSF-ALL. While constructing BCSF-ALL, sorting is performed at each mode to identify the nonzeros belonging to the same fiber and same slice. CSF is then constructed on the sorted tensor. Load balancing is achieved via binning [3], where slices with similar lengths are binned together. To construct MM-CSF, we first collect the fiber lengths of $\geq d$ modes, then create $p$ disjoint partitions of nonzeros, and finally, construct CSF for each partition. There is no binning required for MM-CSF. The available BCSF-ALL implementation of [26] uses an unoptimized sort in its preprocessing step. For an unbiased comparison, we replaced it with an optimized version that is used in MM-CSF preprocessing step. Figure 10 presents the normalized time to construct BCSF-ALL and MM-CSF for 3D tensors, including memory copy time for one iteration (i.e., time taken to copy the data from host to GPU device). We observe that on average, MM-CSF incurs merely $15 \%$ extra preprocessing overhead over BCSF-ALL. Additionally, MM-CSF consumes significantly less space than BCSF-ALL to store the tensor. Since one might need to perform memory copy with each CPD iteration depending on the size of the tensor, MM-CSF will have a significant advantage over BCSF-ALL in such cases.

\subsection{Application speedup}

Figure 11 demonstrates the speedup achieved in CPD computation of $3 \mathrm{D}$ tensors by using MM-CSF as the storage format in conjunction with the optimized MTTKRP kernels. The reported time is an average of ten iterations. Apart from MTTKRP, all the remaining kernels in the application are invocations of CPU BLAS functions. After each MTTKRP iteration, the updated matrix is copied back to the CPU, where it is used as an input by the BLAS kernels, 


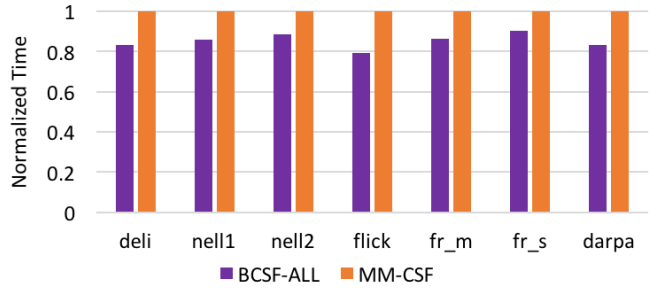

Figure 10: Pre-processing time of BCSF-ALL and MM-CSF

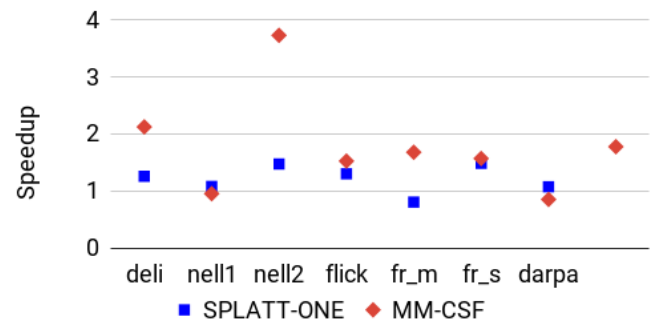

Figure 11: Speedup in CP decomposition using MM-CSF and SPLATT-ONE compared to SPLATT-ALL

followed by a normalization on the column vectors (Line 5 in Algorithm 1). This normalized matrix needs to copied back to GPU to be used in the next MTTKRP computation. Despite the GPU memory copy overhead at each iteration, we outperform SPLATT-ALL from SPLATT by a factor of $1.8 \times$ on average. One of our future endeavor involves replacing the CPU BLAS functions with cuBLAS routines to avoid the back-and-forth memory copy time.

\section{RELATED WORK}

Sparse tensor decompositions and their related operations have attracted attention of researchers to improve their performance and storage. Like matrix factorization [40], [39], [27], tensor factorization is also gaining significant popularity. We briefly discuss prior performance optimization work of MTTKRP operation and CANDECOMP/PARAFAC decomposition (CPD) for sparse tensors.

Tensor Toolbox [5] and Tensorlab [38] packages implement CPD and MTTKRP based on COO format, where an MTTKRP operation is computed as a series of sparse tensor-times-vector. DFacTo [13] performs an MTTKRP by computing multiple sparse matrix-vector multiplication (SpMV) routines which can be computed efficiently through existing high performance libraries. However, the intermediate storage of it could be very large by saving the outputs of SpMV. Smith et al. [29, 35] proposed the CSF storage format, an extension of Compressed Sparse Row (CSR) format for sparse matrices, and optimized the performance and memory access of MTTKRP in the SPLATT library along with the support of different tensor decompositions and completion algorithms [33]. Choi et al. [12] employed two blocking strategies to further optimize MTTKRP using the CSF format. A new Hierarchical COOrdinate (HiCOO) format, derived from the $\mathrm{COO}$ format, was recently proposed by $\mathrm{Li}$ et al. [23]. HiCOO compresses tensor indices as units of sparse blocks, to save storage and to reduce a sparse tensor algorithm's memory footprint. However, HiCOO does not work well for hypersparse tensors, a.k.a. tensors with extreme low density, sometimes even after reordering [24], thus the other formats like CSF and COO still play important roles. Baskaran et al. proposed multiple optimization techniques to address load imbalance, sparsity, etc. of sparse tensor computation in [6-8].

Some research targeted on other platforms. GigaTensor [19] targets on large-scale sparse tensors by providing a scalable framework using the MapReduce paradigm. Blanco et al. [9] accelerated tensor decompositions using a queuing strategy to exploit the dependency and data reuse using Spark engine on distributed platforms. Kaya et al. [20] scaled CPD on distributed memory systems using message passing interface (MPI), the implementation of which is also based on COO format. Smith et al. [34] improved MTTKRP performance on Intel Xeon Phi Knights Landing manycore processor. A Parallel Tensor Infrastructure (ParTI!) supports COO stored tensors to do MTTKRP on NVIDIA GPUs by parallelizing nonzeros and using atomic operations. Liu et al. [25] proposed a more compressed Flagged $\mathrm{COO}$ (F-COO) format uses a fast parallel scan routine on GPUs to reduce write conflicts. However, F-COO closely depends on a particular MTTKRP operation, which affects its flexibility. Nisa et al. [26] optimized MTTKRP performance by proposing loadbalanced data structure (BCSF) and parallel strategies, which makes CSF variant MTTKRPP being efficient on GPUs. Phipps et al. [28] leverages the Kokkos framework [14] to optimize MTTKRP on CPUs and GPUs using a single code implementation. Our work further improves MTTKRP by making CSF and BCSF formats more adaptable and efficient to MTTKRP and CPD.

\section{CONCLUSION}

In recent years, tensors have become mainstream in high-performance computing. Several frameworks and libraries are being developed to optimize operations on sparse tensors. Efficient and compact representations of high-order sparse tensors are crucial on architectures with limited global memory and low energy footprint, like GPUs. In this paper, we devise MM-CSF, a mixed-mode storage format for sparse tensors of arbitrary dimensions. Through extensive evaluation on an NVIDIA Volta GPU, we demonstrate the efficacy of $\mathrm{MM}-\mathrm{CSF}$ in (a) reducing the storage requirement for sparse tensors, and (b) improving the performance of computations like tensor factorizations.

\section{ACKNOWLEDGMENTS}

We thank the reviewers for the valuable feedback and the Ohio Supercomputer Center for use of their GPU resources. This material is based upon work supported by the National Science Foundation under Grant No. 1816793 and 1513120. This research is also partially funded by the US Department of Energy, Office for Advanced Scientific Computing (ASCR) under Award No. 66150: "CENATE: The Center for Advanced Technology Evaluation" and the Laboratory Directed Research and Development program at PNNL under contract No. ND8577. Pacific Northwest National Laboratory (PNNL) is a multiprogram national laboratory operated for DOE by Battelle Memorial Institute under Contract DE-AC05-76RL01830. This research was partially supported by the Exascale Computing Project (ECP), Project Number: 17-SC-20-SC, a collaborative effort of two DOE organizations âĂŞ the Office of Science and the National Nuclear Security Administration. 


\section{REFERENCES}

[1] [n. d.]. nvprof-metrics. https://docs.nvidia.com/cuda/profiler-users-guide/index. html. Accessed: 2018-09-30.

[2] Martín Abadi, Ashish Agarwal, Paul Barham, Eugene Brevdo, Zhifeng Chen, Craig Citro, Greg S Corrado, Andy Davis, Jeffrey Dean, Matthieu Devin, et al 2016. Tensorflow: Large-scale machine learning on heterogeneous distributed systems. arXiv preprint arXiv:1603.04467 (2016).

[3] Arash Ashari, Naser Sedaghati, John Eisenlohr, Srinivasan Parthasarathy, and P Sadayappan. 2014. Fast sparse matrix-vector multiplication on GPUs for graph applications. In Proceedings of the international conference for high performance computing, networking, storage and analysis. IEEE Press, 781-792.

[4] Brett W Bader, Michael W Berry, and Murray Browne. 2008. Discussion tracking in Enron email using PARAFAC. In Survey of Text Mining II. Springer, 147-163.

[5] Brett W. Bader, Tamara G. Kolda, et al. 2015. MATLAB Tensor Toolbox Version 2.6. Available online. http://www.sandia.gov/ tgkolda/TensorToolbox/

[6] M. Baskaran, T. Henretty, B. Pradelle, M. H. Langston, D. Bruns-Smith, J. Ezick, and R. Lethin. 2017. Memory-efficient parallel tensor decompositions. In 2017 IEEE High Performance Extreme Computing Conference (HPEC). 1-7. https://doi. org/10.1109/HPEC.2017.8091026

[7] Muthu Baskaran, Benoit Meister, and Richard Lethin. 2014. Low-overhead Loadbalanced Scheduling for Sparse Tensor Computations. In IEEE High Performance Extreme Computing Conference. Waltham, MA.

[8] Muthu Baskaran, Benoit Meister, Nicolas Vasilache, and Richard Lethin. 2012. Ef ficient and Scalable Computations with Sparse Tensors. In IEEE High Performance Extreme Computing Conference. Waltham, MA.

[9] Zachary Blanco, Bangtian Liu, and Maryam Mehri Dehnavi. 2018. CSTF: LargeScale Sparse Tensor Factorizations on Distributed Platforms. In Proceedings of the 47th International Conference on Parallel Processing (ICPP 2018). ACM, New York, NY, USA, Article 21, 10 pages. https://doi.org/10.1145/3225058.3225133

[10] Aydin Buluc and John R Gilbert. 2008. On the representation and multiplication of hypersparse matrices. In Parallel and Distributed Processing, 2008. IPDPS 2008. IEEE International Symposium on. IEEE, 1-11.

[11] J Douglas Carroll and Jih-Jie Chang. 1970. Analysis of individual differences in multidimensional scaling via an N-way generalization of âĂIJEckart-YoungâĂ decomposition. Psychometrika 35, 3 (1970), 283-319.

[12] Jee Choi, Xing Liu, Shaden Smith, and Tyler Simon. 2018. Blocking Optimization Techniques for Sparse Tensor Computation. In 2018 IEEE International Parallel and Distributed Processing Symposium (IPDPS). IEEE, 568-577.

[13] Joon Hee Choi and S Vishwanathan. 2014. DFacTo: Distributed factorization of tensors. In Advances in Neural Information Processing Systems. 1296-1304.

[14] H Carter Edwards, Christian R Trott, and Daniel Sunderland. 2014. Kokkos: Enabling manycore performance portability through polymorphic memory access patterns. F. Parallel and Distrib. Comput. 74, 12 (2014), 3202-3216.

[15] Richard A Harshman. 1970. Foundations of the PARAFAC procedure: Models and conditions for an" explanatory" multimodal factor analysis. (1970)

[16] Joyce C Ho, Joydeep Ghosh, Steve R Steinhubl, Walter F Stewart, Joshua C Denny, Bradley A Malin, and Jimeng Sun. 2014. Limestone: High-throughput candidate phenotype generation via tensor factorization. fournal of biomedical informatics 52 (2014), 199-211.

[17] Joyce C Ho, Joydeep Ghosh, and Jimeng Sun. 2014. Marble: high-throughput phenotyping from electronic health records via sparse nonnegative tensor factorization. In Proceedings of the 20th ACM SIGKDD international conference on Knowledge discovery and data mining. ACM, 115-124.

[18] Inah Jeon, Evangelos E Papalexakis, U Kang, and Christos Faloutsos. 2015. Haten2: Billion-scale tensor decompositions. In Data Engineering (ICDE), 2015 IEEE 31st International Conference on. IEEE, 1047-1058.

[19] U Kang, Evangelos Papalexakis, Abhay Harpale, and Christos Faloutsos. 2012. Gigatensor: scaling tensor analysis up by 100 times-algorithms and discoveries. In Proceedings of the 18th ACM SIGKDD international conference on Knowledge discovery and data mining. ACM, 316-324.

[20] O. Kaya and B. UÃ ğar. 2015. Scalable Sparse Tensor Decompositions in Distributed Memory Systems. In Proceedings of the International Conference for High Performance Computing, Networking, Storage and Analysis. ACM, 77:1-77:11. https://doi.org/10.1145/2807591.2807624

[21] Tamara G Kolda and Brett W Bader. 2009. Tensor decompositions and applications. SIAM review 51, 3 (2009), 455-500.

[22] Jiajia Li, Yuchen Ma, and Richard Vuduc. 2017. ParTI!: A Parallel Tensor Infrastructure for Multicore CPU and GPUs. Available from https://github.com/ hpcgarage/ParTI.

[23] Jiajia Li, Jimeng Sun, and Richard Vuduc. 2018. HiCOO: Hierarchical Storage of Sparse Tensors. In Proceedings of the International Conference for High Performance Computing, Networking, Storage and Analysis (SC '18). ACM, New York, NY, USA.

[24] Jiajia Li, Bora Uçar, Umit V. Çatalyürek, Jimeng Sun, Kevin Barker, and Richard Vuduc. 2019. Efficient and Effective Sparse Tensor Reordering. In Proceedings of the ACM International Conference on Supercomputing (ICS '19). ACM, New York, NY, USA, 227-237. https://doi.org/10.1145/3330345.3330366

[25] Bangtian Liu, Chengyao Wen, Anand D Sarwate, and Maryam Mehri Dehnavi. 2017. A Unified Optimization Approach for Sparse Tensor Operations on GPUs. In Cluster Computing (CLUSTER), 2017 IEEE International Conference on. IEEE, 47-57.

[26] Israt Nisa, Jiajia Li, Aravind Sukumaran-Rajam, Richard W. Vuduc, and P. Sadayappan. 2019. Load-Balanced Sparse MTTKRP on GPUs. In 2019 IEEE International Parallel and Distributed Processing Symposium (IPDPS), Vol. abs/1904.03329.

[27] Israt Nisa, Aravind Sukumaran-Rajam, Rakshith Kunchum, and P Sadayappan. 2017. Parallel ccd++ on gpu for matrix factorization. In Proceedings of the General Purpose GPUs. ACM, 73-83.

[28] Eric T Phipps and Tamara G Kolda. 2019. Software for Sparse Tensor Decomposition on Emerging Computing Architectures. SIAM fournal on Scientific Computing 41, 3 (2019), C269-C290.

[29] Shaden Smith. 2019. Algorithms for Large-Scale Sparse Tensor Factorization. Ph.D. Dissertation. University of Minnesota, Minneapolis, MN, USA. http://hdl.handle. net/11299/206375

[30] Shaden Smith, Jee W. Choi, Jiajia Li, Richard Vuduc, Jongsoo Park, Xing Liu, and George Karypis. 2017. FROSTT: The Formidable Repository of Open Sparse Tensors and Tools. http://frostt.io/

[31] Shaden Smith and George Karypis. 2015. Tensor-matrix products with a compressed sparse tensor. In Proceedings of the 5th Workshop on Irregular Applications: Architectures and Algorithms. ACM, 5.

[32] Shaden Smith and George Karypis. 2016. A medium-grained algorithm for sparse tensor factorization. In Parallel and Distributed Processing Symposium, 2016 IEEE International. IEEE, 902-911.

[33] Shaden Smith, Jongsoo Park, and George Karypis. 2016. An Exploration of Optimization Algorithms for High Performance Tensor Completion. Proceedings of the 2016 ACM/IEEE conference on Supercomputing (2016)

[34] Shaden Smith, Jongsoo Park, and George Karypis. 2017. Sparse Tensor Factorization on Many-Core Processors with High-Bandwidth Memory. 31st IEEE International Parallel \& Distributed Processing Symposium (IPDPS'17) (2017).

[35] Shaden Smith, Niranjay Ravindran, Nicholas D Sidiropoulos, and George Karypis. 2015. SPLATT: Efficient and parallel sparse tensor-matrix multiplication. In Parallel and Distributed Processing Symposium (IPDPS), 2015 IEEE International. IEEE, 61-70.

[36] Jimeng Sun, Spiros Papadimitriou, and S Yu Philip. 2006. Window-based Tensor Analysis on High-dimensional and Multi-aspect Streams.. In ICDM, Vol. 6. 10761080.

[37] Jimeng Sun, Dacheng Tao, and Christos Faloutsos. 2006. Beyond streams and graphs: dynamic tensor analysis. In Proceedings of the 12th ACM SIGKDD international conference on Knowledge discovery and data mining. ACM, 374-383.

[38] Nico Vervliet, Otto Debals, and Lieven De Lathauwer. 2016. Tensorlab 3.0âĂŤNumerical optimization strategies for large-scale constrained and coupled matrix/tensor factorization. In Signals, Systems and Computers, 2016 50th Asilomar Conference on. IEEE, 1733-1738.

[39] Hsiang-Fu Yu, Cho-Jui Hsieh, Si Si, and Inderjit S Dhillon. 2014. Parallel matrix factorization for recommender systems. Knowledge and Information Systems 41, 3 (2014), 793-819.

[40] Yunhong Zhou, Dennis Wilkinson, Robert Schreiber, and Rong Pan. 2008. Largescale parallel collaborative filtering for the netflix prize. In International conference on algorithmic applications in management. Springer, 337-348. 


\section{Appendix: Artifact Description/Artifact Evaluation}

\begin{abstract}
SUMMARY OF THE EXPERIMENTS REPORTED
The results for GPU are collected on an NVIDIA Volta V100 GPU with 16GB memory. The results for CPU are collected using a Dell PowerEdge R740 two-socket servers with Intel Xeon 6148. For the GPU codes, NVCC-9.2 compiler is used, and for the CPU code gcc (GCC) 7.3.0 is used with the OpenMP flag. Number of threads is set to 40 .
\end{abstract}

\section{ARTIFACT AVAILABILITY}

Software Artifact Availability: All author-created software artifacts are maintained in a public repository under an OSI-approved license.

Hardware Artifact Availability: There are no author-created hardware artifacts.

Data Artifact Availability: All author-created data artifacts are maintained in a public repository under an OSI-approved license.

Proprietary Artifacts: None of the associated artifacts, authorcreated or otherwise, are proprietary.

List of URLs and/or DOIs where artifacts are available:

10.5281/zenodo. 3379102

https://github.com/isratnisa/MM-CSF

\section{BASELINE EXPERIMENTAL SETUP, AND MODIFICATIONS MADE FOR THE PAPER}

Relevant hardware details: Volta V100 GPU, Intel(R) Xeon(R) Gold $6148 \mathrm{CPU}$

Operating systems and versions: Red Hat Enterprise Linux Server VERSION $=7.5$

Compilers and versions: NVCC 9.2, gcc (GCC) 7.3.0

Libraries and versions: boost 1.67, OpenBLAS-3.6, openMP

Key algorithms: MTTKRP, CPD

Input datasets and versions: http://frostt.io

Output from scripts that gathers execution environment information.

LMOD_FAMILY_COMPILER_VERSION $=18.0 .3$

MKLROOT=/opt/intel/18.0.3/compilers_and_libraries_20」 $\hookrightarrow \quad 18 /$ linux/mkl
MANPATH=/opt/mvapich2/intel/18.0/2.3/share/man:/opt/ $\hookrightarrow$ intel/18.0.3/itac_latest/man:/opt/intel/18.0.3/d

$\hookrightarrow$ ocumentation_2018/en/debugger/gdb-igfx/man:/opt/ .

$\hookrightarrow$ intel/18.0.3/inspector_2018/man:/opt/intel/18.0.」

$\hookrightarrow$ 3/compilers_and_libraries_2018/linux/man/common: 」

$\hookrightarrow$ /opt/torque/share/man:/opt/moab/man:/apps/lmod/1」

$\hookrightarrow \mathrm{mod} / \mathrm{share} / \mathrm{man}$ :/usr/share/man/overrides:/usr/shar 」

$\hookrightarrow$ e/man:/usr/local/share/man:/opt/ibutils/share/ma 」

$\hookrightarrow \mathrm{n}$ :/opt/ddn/ime/share/man:/opt/puppetlabs/puppet/

$\hookrightarrow$ share/man:/opt/intel/18.0.3/vtune_amplifier/man:

$\hookrightarrow$ /opt/intel/18.0.3/advisor/man

__LMOD_REF_COUNT_FPATH=/opt/intel/18.0.3/compilers_a 」

$\hookrightarrow$ nd_libraries_2018/linux/mkl/include: 1

XALT_ETC_DIR=/apps/xalt/etc

_ModuleTable003_=ZE9yZGVyIl09NCxwcm9wVD17fSxbInN0YWN 」

$\hookrightarrow$ rRGVwdGgiXT0xLFsic3RhdHVzIl09ImFjdGl2ZSIsWyJ1c2V 」

$\hookrightarrow$ yTmFtZSJdPSJtdmFwaWNoMi IsfSx4YWxOPXtbImZuI109I i 」

$\hookrightarrow$ hcHBzL2xtb2RmaWx lcy9Db3J1L3hhbHQvbGF0ZXN0Lmx1YSI

$\hookrightarrow$ sWyJmdWxsTmFtZSJdPSJ4YWx0L2xhdGVzdCIsWyJsb2FkT3J

$\hookrightarrow$ kZXIiXT0xLHByb3BUPXt9LF sic3RhY2tEZXB0aCJdPTEsWyJ

$\hookrightarrow \quad$ zdGF0dXMiXT0iYWN0aXZlIixbInVzZXJOYW11Il09InhhbHQ

$\hookrightarrow \quad$ iLHOsfSXtcGFOaEE9eyIvYXBwcy9sbW9kZmlsZXMvTVBJL21

$\hookrightarrow$ udGVsLzE4LjAvbXZhcGljaDIvMi4zIiwiL2FwcHMvbG1vZGZ

$\hookrightarrow \quad$ pbGVZLONvbXBpbGVyL2ludGVsLzE4LjAiLCIvYXBwcy9sbW9 」

$\hookrightarrow$ kZmlsZXMvTGludXgiLCIvYXBwcy9sbW9kZmlsZXMvQ29y

PBS_VERSION=TORQUE-6.1.2

MPI_FFLAGS=-I/opt/mvapich2/intel/18.0/2.3/include

IPPROOT=/opt/intel/18.0.3/compilers_and_libraries_20 」

$\hookrightarrow$ 18/linux/ipp

MPICH_HOME=/opt/mvapich2/intel/18.0/2.3

MPI_F90FLAGS=-I/opt/mvapich2/intel/18.0/2.3/include

$\mathrm{F} 90=\mathrm{i}$ fort

SHELL=/bin/bash

TERM=xterm-256color

__LMOD_REF_COUNT_MODULEPATH=/apps/lmodfiles/MPI/inte

$\hookrightarrow \quad 1 / 18.0 /$ mvapich2/2.3:1;/apps/lmodfiles/Compiler/i

$\hookrightarrow$ ntel/18.0:1;/apps/lmodfiles/Linux:1;/apps/lmodfi

$\hookrightarrow$ les/Core:1;/apps/lmod/lmod/modulefiles/Core: 1

HISTSIZE $=1000$

PBS_JOBNAME $=$ STDIN

MODULEPATH_ROOT=/apps/lmodfiles

TMPDIR $=/$ tmp/pbstmp .472725

LMOD_SYSTEM_DEFAULT_MODULES=modules

XALT_EXECUTABLE_TRACKING=yes

LMOD_PACKAGE_PATH=/apps/lmodfiles/site 
LIBRARY_PATH=/opt/intel/18.0.3/compilers_and_librari 」 $\hookrightarrow$ es_2018/linux/linux/lib/intel64_lin:/opt/intel/1 」

$\hookrightarrow$ 8.0.3/compilers_and_libraries_2018/linux/daal/li

$\hookrightarrow$ b/intel64_lin:/opt/intel/18.0.3/compilers_and_li 」

$\hookrightarrow$ braries_2018/linux/ipp/lib/intel64_lin:/opt/inte」

$\hookrightarrow \quad 1 / 18.0 .3 /$ compilers_and_libraries_2018/linux/mkl/」

$\hookrightarrow$ lib/intel64_lin:/opt/intel/18.0.3/compilers_and_」

$\hookrightarrow$ libraries_2018/linux/tbb/lib/intel64_lin/gcc4.4

__LMOD_REF_COUNT_COMPILER_PATH=/apps/xalt/xalt/bin: 1

LD_PRELOAD=/apps/xalt/xalt/lib64/libxalt_init.so

PBS_ENVIRONMENT=PBS_INTERACTIVE

LMOD_PKG=/apps $/ 1 \mathrm{mod} / 1 \mathrm{mod}$

FPATH=/opt/intel/18.0.3/compilers_and_libraries_2018」

$\hookrightarrow /$ linux/mkl/include

COMPILER=intel

QTDIR=/usr/lib64/qt-3.3

IMEDIR=

LMOD_VERSION $=7.8$

QTINC=/usr/lib64/qt-3.3/include

PBS_HOME=/var/spool/torque

$C C=i C C$

__LMOD_REF_COUNT_LOADEDMODULES $=x a l t /$ latest $: 1 ; \mathrm{cxx} 17 / 7$ 」

$\hookrightarrow \quad .3 .0: 1$; intel/18.0.3:1;mvapich2/2.3:1;modules/au2 」

$\hookrightarrow \quad 018: 1$

QT_GRAPHICSSYSTEM_CHECKED $=1$

INTEL_DIR=/opt/intel/18.0.3

USER $=$ USER

PBS_TASKNUM $=1$

MV2_CPU_BINDING_POLICY=hybrid

COMPILER_MINOR $=0$
LS_COLORS $=r s=0: d i=38 ; 5 ; 27: \ln =38 ; 5 ; 51: \mathrm{mh}=44 ; 38 ; 5 ; 15: \mathrm{p}_{\text {」 }}$

$\hookrightarrow \quad \mathrm{i}=40 ; 38 ; 5 ; 11: \mathrm{so}=38 ; 5 ; 13: \mathrm{do}=38 ; 5 ; 5: \mathrm{bd}=48 ; 5 ; 232 ; 38$ 」

$\hookrightarrow \quad ; 5 ; 11: \mathrm{cd}=48 ; 5 ; 232 ; 38 ; 5 ; 3:$ or $=48 ; 5 ; 232 ; 38 ; 5 ; 9: \mathrm{mi}=0$ 」

$\hookrightarrow \quad 5 ; 48 ; 5 ; 232 ; 38 ; 5 ; 15: \mathrm{su}=48 ; 5 ; 196 ; 38 ; 5 ; 15: \mathrm{sg}=48 ; 5 ; 1$ 」

$\hookrightarrow \quad 1 ; 38 ; 5 ; 16: \mathrm{ca}=48 ; 5 ; 196 ; 38 ; 5 ; 226: \mathrm{tw}=48 ; 5 ; 10 ; 38 ; 5 ; 1$ 」

$\hookrightarrow \quad 6: \mathrm{ow}=48 ; 5 ; 10 ; 38 ; 5 ; 21: \mathrm{st}=48 ; 5 ; 21 ; 38 ; 5 ; 15: \mathrm{ex}=38 ; 5 ;$ 」

$\hookrightarrow \quad 34: * . \operatorname{tar}=38 ; 5 ; 9: * . \operatorname{tgz}=38 ; 5 ; 9: * . \operatorname{arc}=38 ; 5 ; 9: * . \operatorname{arj}=$ 」

$\hookrightarrow \quad 38 ; 5 ; 9: *$. taz $=38 ; 5 ; 9: * .1 \mathrm{ha}=38 ; 5 ; 9: * .1 \mathrm{z} 4=38 ; 5 ; 9: *$.

$\hookrightarrow \quad l z h=38 ; 5 ; 9: * .1 z m a=38 ; 5 ; 9: * . t l z=38 ; 5 ; 9: * . t x z=38 ; 5$ 」

$\hookrightarrow \quad ; 9: * . t z o=38 ; 5 ; 9: * . t 7 z=38 ; 5 ; 9: * . z i p=38 ; 5 ; 9: * . z=38$ ।

$\hookrightarrow \quad ; 5 ; 9: * . z=38 ; 5 ; 9: * . d z=38 ; 5 ; 9: * . g z=38 ; 5 ; 9: * .1 r z=38$ ।

$\hookrightarrow \quad ; 5 ; 9: * .1 z=38 ; 5 ; 9: * .1 z 0=38 ; 5 ; 9: * . x z=38 ; 5 ; 9: * . b z 2=$ 」

$\hookrightarrow \quad 38 ; 5 ; 9: * . b z=38 ; 5 ; 9: * . t b z=38 ; 5 ; 9: * . t b z 2=38 ; 5 ; 9: *$. 」

$\hookrightarrow \quad \mathrm{tz}=38 ; 5 ; 9: * . \mathrm{deb}=38 ; 5 ; 9: * . \mathrm{rpm}=38 ; 5 ; 9: * . \mathrm{jar}=38 ; 5 ; 9$ ।

$\hookrightarrow \quad: *$.war $=38 ; 5 ; 9: *$.ear $=38 ; 5 ; 9: *$. sar $=38 ; 5 ; 9: *$. rar $=38$

$\hookrightarrow \quad ; 5 ; 9: *$.alz $=38 ; 5 ; 9: *$. ace $=38 ; 5 ; 9: * . z 00=38 ; 5 ; 9: * . c p$ ।

$\hookrightarrow \quad$ io $=38 ; 5 ; 9: * .7 z=38 ; 5 ; 9: * . r z=38 ; 5 ; 9: * . c a b=38 ; 5 ; 9: *$ 」

$\hookrightarrow \quad . j p g=38 ; 5 ; 13: * . j p e g=38 ; 5 ; 13: *$.gif $=38 ; 5 ; 13: * . b m p=$ 」

$\hookrightarrow \quad 38 ; 5 ; 13: *$. pbm=38;5;13:*.pgm=38;5;13:*.ppm=38;5;1 」

$\hookrightarrow \quad 3: *$. tga $=38 ; 5 ; 13: * . x b m=38 ; 5 ; 13: * . x p m=38 ; 5 ; 13: * . t i$ 」

$\hookrightarrow \mathrm{f}=38 ; 5 ; 13: *$.tiff $=38 ; 5 ; 13: *$.png $=38 ; 5 ; 13: *$.svg $=38$; ।

$\hookrightarrow 5 ; 13: *$. svgz $=38 ; 5 ; 13: * . \mathrm{mng}=38 ; 5 ; 13: * . p c x=38 ; 5 ; 13:$,

$\hookrightarrow \quad$ *.mov $=38 ; 5 ; 13: * . \mathrm{mpg}=38 ; 5 ; 13: *$.mpeg $=38 ; 5 ; 13: * . \mathrm{m} 2 \mathrm{v}$ ।

$\hookrightarrow \quad=38 ; 5 ; 13: * . \mathrm{mkv}=38 ; 5 ; 13: *$. webm $=38 ; 5 ; 13: *$. ogm $=38 ; 5$ 」

$\hookrightarrow \quad ; 13: * . \mathrm{mp} 4=38 ; 5 ; 13: * . \mathrm{m} 4 \mathrm{v}=38 ; 5 ; 13: * . \mathrm{mp} 4 \mathrm{v}=38 ; 5 ; 13: *$ 」

$\hookrightarrow \quad$ vob $=38 ; 5 ; 13: *$. qt $=38 ; 5 ; 13: *$. nuv $=38 ; 5 ; 13: *$. wmv $=38$ 」

$\hookrightarrow \quad ; 5 ; 13: *$. asf $=38 ; 5 ; 13: * . r m=38 ; 5 ; 13: * . r m v b=38 ; 5 ; 13:$ ।

$\hookrightarrow \quad * . f l c=38 ; 5 ; 13: *$ avi $=38 ; 5 ; 13: * . f l i=38 ; 5 ; 13: * . f l v=$ ।

$\hookrightarrow \quad 38 ; 5 ; 13: * . g l=38 ; 5 ; 13: * . d l=38 ; 5 ; 13: * . x c f=38 ; 5 ; 13:$ 」

$\hookrightarrow \quad * . x w d=38 ; 5 ; 13: *$. yuv $=38 ; 5 ; 13: *$. cgm $=38 ; 5 ; 13: *$. emf $=$ 」

$\hookrightarrow \quad 38 ; 5 ; 13: *$. axv $=38 ; 5 ; 13: *$. an $x=38 ; 5 ; 13: *$.ogv $=38 ; 5 ; 1$ 」

$\hookrightarrow \quad 3: *$. og $x=38 ; 5 ; 13: *$. aac $=38 ; 5 ; 45: * . a u=38 ; 5 ; 45: * . f l a$ ।

$\hookrightarrow \mathrm{c}=38 ; 5 ; 45: *$. mid $=38 ; 5 ; 45: *$.midi $=38 ; 5 ; 45: *$.mka $=38 ;$ ।

$\hookrightarrow \quad 5 ; 45: *$.mp3 $=38 ; 5 ; 45: * . \mathrm{mpc}=38 ; 5 ; 45: *$. ogg $=38 ; 5 ; 45: *$ 」

$\hookrightarrow \quad . r a=38 ; 5 ; 45: *$.wav $=38 ; 5 ; 45: * . a x a=38 ; 5 ; 45: *$. oga $=38$ 」

$\hookrightarrow \quad ; 5 ; 45: *$. spx $=38 ; 5 ; 45: * . x s p f=38 ; 5 ; 45:$

LMOD_sys $=$ Linux

LD_LIBRARY_PATH=/opt/mvapich2/intel/18.0/2.3/lib:/ap 」

$\hookrightarrow$ ps/gnu/7.3.0/lib64:/apps/gnu/7.3.0/lib:/opt/inte 」

$\hookrightarrow$ 1/18.0.3/debugger_2018/1ibipt/intel64/lib:/opt/i 」

$\hookrightarrow$ ntel/18.0.3/compilers_and_libraries_2018/linux/1」

$\hookrightarrow$ ib/intel64_lin:/opt/intel/18.0.3/compilers_and_l」

$\hookrightarrow$ ibraries_2018/linux/daal/lib/intel64_lin:/opt/in 」

$\hookrightarrow$ tel/18.0.3/compilers_and_libraries_2018/linux/ip ।

$\hookrightarrow$ p/lib/intel64_lin:/opt/intel/18.0.3/compilers_an」

$\hookrightarrow$ d_libraries_2018/linux/mkl/lib/intel64_lin:/opt/」

$\hookrightarrow$ intel/18.0.3/compilers_and_libraries_2018/linux/」

$\hookrightarrow$ tbb/lib/intel64_lin/gcc4.4:/opt/torque/lib64:/op」

$\hookrightarrow \quad t /$ torque/lib: :

$X X X \_C O M P I L E R \_M A J O R=18$

$X X X \_F A M I L Y \_M P I=m v a p i c h 2$

PBSCOREDUMP $="$ "

CPATH=/opt/intel/18.0.3/compilers_and_libraries_2018」

$\hookrightarrow /$ linux/mkl/include:/opt/intel/18.0.3/compilers_a」

$\hookrightarrow$ nd_libraries_2018/linux/tbb/include 
_ModuleTable004_=ZSIsIi9hcHBzL2xtb2QvbG1vZC9tb2R1bGV $\hookrightarrow$ maWxlcy9Db3J1Iix9LFsic3lzdGVtQmFzZU1QQVRIII09Ii9 」

$\hookrightarrow$ hcHBzL2xtb2RmaWx lcy9MaW51eDovYXBwcy9sbW9kZmlsZXM」

$\hookrightarrow \quad$ VQ29yZTovYXBwcy9sbW9kL2xtb2QvbW9kdWXIZmlsZXMvQ29」

$\hookrightarrow \quad \mathrm{yZSISfQ}==$

PBS_WALLTIME $=10740$

$X X X \_C X X=i C P C$

_LLMOD_REF_COUNT__LMFILES_=/apps/lmodfiles/Core/xalt 」

$\hookrightarrow$ /latest.lua:1;/apps/lmodfiles/Compiler/intel/18.

$\hookrightarrow \quad 0 /$ cxx17/7.3.0.lua: 1 ;/apps/lmodfiles/Core/intel/1

$\hookrightarrow$ 8.0.3.lua:1;/apps/lmodfiles/Compiler/intel/18.0/।

$\hookrightarrow$ mvapich2/2.3.lua:1;/apps/lmodfiles/Core/modules/」

$\hookrightarrow$ au2018.lua:1

PBS_MOMPORT $=15003$

PBS_GPUFILE=/var/spool/torque/aux//472725. gpu

LMOD_SITE_NAME $=X X X$

MPIEXEC_COMM=pmi

PBS_O_QUEUE=batch

LMOD_PREPEND_BLOCK=normal

LMOD_FAMILY_MPI_VERSION $=2.3$

MPI_CFLAGS=-I/opt/mvapich2/intel/18.0/2.3/include

MPI_CXXFLAGS=-I/opt/mvapich2/intel/18.0/2.3/include

MPI_LIBS=-L/opt/mvapich2/intel/18.0/2.3/1ib -lmpich

$\hookrightarrow \quad$-libverbs -lpthread

NLSPATH=/opt/intel/18.0.3/debugger_2018/gdb/intel64/

$\hookrightarrow$ share/locale/\%1_\%t/\%N:/opt/intel/18.0.3/compiler」

$\hookrightarrow$ S_and_libraries_2018/linux/lib/intel64_lin/local」

$\hookrightarrow$ e/\%1_\%t/\% : /opt/intel/18.0.3/compilers_and_libra」

$\hookrightarrow$ ries_2018/linux/mkl/lib/intel64_lin/locale/\%1_\%t 」

$\hookrightarrow / \% \mathrm{~N}$

PATH=/apps/xalt/xalt/bin:/opt/mvapich2/intel/18.0/2. J

$\hookrightarrow$ 3/bin:/apps/gnu/7.3.0/bin:/opt/intel/18.0.3/itac」

$\hookrightarrow$ _latest/bin:/opt/intel/18.0.3/advisor/bin64:/opt 」

$\hookrightarrow$ /intel/18.0.3/vtune_amplifier/bin64:/opt/intel/1」

$\hookrightarrow$ 8.0.3/inspector_2018/bin64:/opt/intel/18.0.3/com」

$\hookrightarrow$ pilers_and_libraries_2018/linux/bin/intel64:/app

$\hookrightarrow$ s/software_usage:/opt/torque/bin:/usr/lib64/qt-3」

$\hookrightarrow$.3/bin:/opt/XXX/bin:/opt/moab/bin:/bin:/usr/bin:」

$\hookrightarrow$ /usr/local/bin:/usr/local/sbin:/usr/sbin:/opt/ib」

$\hookrightarrow$ utils/bin:/opt/ddn/ime/bin:/opt/puppetlabs/bin

PBS_O_LOGNAME=USER

MAIL=/var/spool/mail/USER

__LMOD_REF_COUNT_NLSPATH=/opt/intel/18.0.3/debugger_ 」

$\hookrightarrow$ 2018/gdb/intel64/share/locale/\%1_\%t/\%N:1;/opt/in」

$\hookrightarrow$ tel/18.0.3/compilers_and_libraries_2018/linux/li J

$\hookrightarrow$ b/intel64_lin/locale/\%1_\%t/\%N:1;/opt/intel/18.0.」

$\hookrightarrow$ 3/compilers_and_libraries_2018/linux/mkl/lib/int 」

$\hookrightarrow$ el64_lin/locale/\%1_\%t/\%N:1
_ModuleTable001_=X01vZHVsZVRhYmxlXz17WyJNVHZlcnNpb24

$\hookrightarrow \quad$ iXT0ZLFsiY19yZWJ1aWxkVG1tZSJdPTg2NDAwLFsiY19zaG9

$\hookrightarrow \quad$ ydFRpbWUiXT1mYWXzZSxkZXB0aFQ9e30sZmFtaWx5PXtbImN

$\hookrightarrow \quad$ vbXBpbGVyIl09ImludGVsIixbIm1waSJdPSJtdmFwaWNoMi I

$\hookrightarrow$ sfSxtVD17Y3h4MTc9e1siZm4iXT0iL2FwcHMvbG1vZGZpbGV 」

$\hookrightarrow \quad$ zLONvbXBpbGVyL2ludGVsLzE4LjAvY3h4MTcvNy4zLjAubHV 」

$\hookrightarrow$ hIixbImZ1bGx0YW11I109ImN4eDE3LzcuMy4wIixbImxvYWR

$\hookrightarrow$ PcmRlciJdPTIscHJvcFQ9e30sWyJzdGFja0RlcHRoIl09Mix 」

$\hookrightarrow$ bInNOYXR1cyJdPSJhY3RpdmUiLFsidXNlck5hbWUiXT0iY3h

$\hookrightarrow \quad 4 M T$ ciLH0saW50ZWw9e1siZm4iXT0iL2FwcHMvbG1vZGZpbGV」

$\hookrightarrow \quad$ zLONvcmUvaW50ZWwvMTguMC4zLmx1YSIsWyJmdWxsTmFt

PBS_0_LANG=en_US.UTF-8

PBS_JOBC00KIE=8DD3E1D6A361CA28088AEFA814243CC6

LMOD_SETTARG_CMD $=$ :

$X X X \_F A M I L Y \_C O M P I L E R=i n t e l$

XXX_MVAPICH2_DIR=/opt/mvapich2/intel/18.0/2.3

TBBROOT=/opt/intel/18.0.3/compilers_and_libraries_20

$\hookrightarrow 18 /$ linux/tbb

PDSH_RCMD_TYPE=ssh

_LMFILES_=/apps/lmodfiles/Core/xalt/latest.lua:/apps」

$\hookrightarrow / 1$ modfiles/Compiler/intel/18.0/cxx17/7.3.0.lua:/ 」

$\hookrightarrow$ apps/lmodfiles/Core/intel/18.0.3.lua:/apps/lmodf 」

$\hookrightarrow$ iles/Compiler/intel/18.0/mvapich2/2.3.lua:/apps/」

$\hookrightarrow$ lmodfiles/Core/modules/au2018.lua

LANG=en_US.UTF-8

PBS_NODENUM $=0$

MODULEPATH=/apps/lmodfiles/MPI/intel/18.0/mvapich2/2 」

$\hookrightarrow \quad .3:$ /apps/lmodfiles/Compiler/intel/18.0:/apps/lmo

$\hookrightarrow$ dfiles/Linux:/apps/lmodfiles/Core:/apps/lmod/lmo 」

$\hookrightarrow d /$ modulefiles/Core

MOABHOMEDIR=/ $\mathrm{var} / \mathrm{spool} / \mathrm{moab}$

$X X X \_F A M I L Y \_C O M P I L E R \_V E R S I O N=18.0 .3$

PBS_NUM_NODES $=1$

KDEDIRS $=/$ usr

LOADEDMODULES $=x a l t /$ latest $: c x x 17 / 7.3 .0:$ intel/18.0.3: $\mathrm{m}_{\text {」 }}$ $\hookrightarrow \quad$ vapich2/2.3:modules/au2018

_ModuleTable_Sz_=4

PBS_0_SHELL=/bin/bash

XXX_MVAPICH2_LIB=/opt/mvapich2/intel/18.0/2.3/lib

LMOD_CMD=/apps $/ 1 \mathrm{mod} / 1 \mathrm{mod} / 1 \mathrm{ibexec} / 1 \mathrm{mod}$

XXX_MPI_CC=mpicc

PBS_JOBID $=472725$.

LMOD_AVAIL_STYLE=system

DAALROOT=/opt/intel/18.0.3/compilers_and_libraries_2 」

$\hookrightarrow$ 018/linux/daal

HISTCONTROL=ignoredups

SSH_ASKPASS=/usr/libexec/openssh/gnome-ssh-askpass

ENVIRONMENT $=$ BATCH

INTEL_PYTHONHOME=/opt/intel/18.0.3/debugger_2018/pyt 」

$\hookrightarrow$ hon/intel64

XXX_F77=ifort

SHLVL=2

$X X X \_F C=i$ fort 
XXX_MPI_CXX=mpic++

__LMOD_REF_COUNT_PATH=/apps/xalt/xalt/bin: 1 ;/opt/mva 」

$\hookrightarrow$ pich2/intel/18.0/2.3/bin:1;/apps/gnu/7.3.0/bin:1」

$\hookrightarrow \quad$;/opt/intel/18.0.3/itac_latest/bin:1;/opt/intel/」

$\hookrightarrow$ 18.0.3/advisor/bin64:1;/opt/intel/18.0.3/vtune_a」

$\hookrightarrow$ mplifier/bin64:1;/opt/intel/18.0.3/inspector_201」

$\hookrightarrow$ 8/bin64:1;/opt/intel/18.0.3/compilers_and_librar 」

$\hookrightarrow$ ies_2018/linux/bin/intel64:1;/apps/software_usag」

$\hookrightarrow \mathrm{e}: 1 ; /$ opt/torque/bin:1;/usr/lib64/qt-3.3/bin:1;/o 」

$\hookrightarrow \mathrm{pt} / \mathrm{XXX} / \mathrm{bin}: 1$;/opt/moab/bin:1;/bin:1;/usr/bin:1;/ J

$\hookrightarrow$ usr/local/bin:1;/usr/local/sbin:1;/usr/sbin:1;/o,

$\hookrightarrow$ pt/ibutils/bin:1;/opt/ddn/ime/bin:1;/opt/puppetl」

$\hookrightarrow$ abs/bin: 1

__LMOD_REF_COUNT_CPATH=/opt/intel/18.0.3/compilers_a 」 $\hookrightarrow$ nd_libraries_2018/linux/mkl/include:1;/opt/intel」

$\hookrightarrow / 18.0 .3 /$ compilers_and_libraries_2018/linux/tbb/i 」 $\hookrightarrow$ nclude: 1

_ModuleTable002_=ZSJdPSJpbnRlbC8xOC4wLjMiLFsibG9hZE9 」 $\hookrightarrow$ yZGVyI109Myxwcm9wVD17fSxbInNOYWNrRGVwdGgiXTOxLFs 」

$\hookrightarrow \quad$ ic3RhdHVzI109ImFjdG12ZSIsWyJ1c2VyTmFtZSJdPSJpbnR 」

$\hookrightarrow \quad$ lbCIsfSxtb2R1bGVzPXtbImZuI109Ii9hcHBzL2xtb2RmaWx

$\hookrightarrow \quad$ lcy9Db3J1L21vZHVsZXMvYXUyMDE4Lmx1YSIsWy JmdWxsTmF ।

$\hookrightarrow$ tZSJdPSJtb2R1bGVZL2F1MjAxOCIsWyJsb2FkT3JkZXIiXT0,

$\hookrightarrow$ 1LHByb3BUPXt9LFsic3RhY2tEZXB0aCJdPTAsWyJzdGF0dXM 」

$\hookrightarrow \quad$ iXT0iYWNOaXZ1IixbInVZZXJOYW11I109Im1 vZHVsZXMiLH0,

$\hookrightarrow$ sbXZhcGljaDI9e1siZm4iXT0iL2FwcHMvbG1vZGZpbGVzLON

$\hookrightarrow \quad$ vbXBpbGVyL2ludGVsLzE4LjAvbXZhcGljaDIvMi4zLmX1YSI 」

$\hookrightarrow \quad$ sWyJmdWxsTmFtZSJdPSJtdmFwaWNoMi 8yLjMiLFsibG9h

__LMOD_REF_COUNT_INCLUDE=/opt/intel/18.0.3/compilers 」

$\hookrightarrow$ _and_libraries_2018/linux/daal/include:1;/opt/in

$\hookrightarrow$ tel/18.0.3/compilers_and_libraries_2018/linux/ip」

$\hookrightarrow \mathrm{p} /$ include:1;/opt/intel/18.0.3/compilers_and_libr」

$\hookrightarrow$ aries_2018/linux/mkl/include: 1

XALT_SCALAR_AND_SPSR_SAMPLING=yes

PBS_VNODENUM $=0$

BASH_ENV=/apps $/ 1 \mathrm{mod} / 1 \mathrm{mod} /$ init $/$ bash

XXX_MPI_FC=mpifort

LOGNAME $=$ USER

LMOD_arch $=x 86 \_64$

MV2_IBA_HCA=ml $x 5 \_0$

CVS_RSH=ssh

QTLIB=/usr/lib64/qt-3.3/lib

XXX_FAMILY_MPI_VERSION $=2.3$

PBS_QUEUE=serial

PDSH_SSH_ARGS_APPEND=-oStrictHostKeyChecking=no

$\hookrightarrow \quad$-oUserKnownHostsFile=/dev/null -oLogLevel=ERROR

MODULESHOME $=/$ apps $/ 1 \mathrm{mod} / 1 \mathrm{mod}$
__LMOD_REF_COUNT_LIBRARY_PATH=/opt/intel/18.0.3/comp $\hookrightarrow$ ilers_and_libraries_2018/linux/linux/lib/intel64 」 $\hookrightarrow \quad$ lin:1;/opt/intel/18.0.3/compilers_and_libraries 」 $\hookrightarrow$ _2018/linux/daal/lib/intel64_lin:1;/opt/intel/18 」

$\hookrightarrow \quad .0 .3 /$ compilers_and_libraries_2018/linux/ipp/lib/

$\hookrightarrow \quad$ intel64_lin:1;/opt/intel/18.0.3/compilers_and_li

$\hookrightarrow$ braries_2018/linux/mkl/lib/intel64_lin:1;/opt/in」

$\hookrightarrow$ tel/18.0.3/compilers_and_libraries_2018/linux/tb」

$\hookrightarrow$ b/lib/intel64_lin/gcc4.4:1

PBS_0_MAIL=/var/spool/mail/USER

PBS_O_SUBMIT_FILTER=/usr/local/sbin/torque_submitfil 」 $\hookrightarrow$ ter

LESSOPEN=||/usr/bin/lesspipe.sh \%s

LMOD_SETTARG_FULL_SUPPORT $=$ no

COMPILER_PATH=/apps $/ \mathrm{xalt} / \mathrm{xalt} / \mathrm{bin}$

_LMOD_REF_COUNT_LD_LIBRARY_PATH=/opt/mvapich2/intel」

$\hookrightarrow / 18.0 / 2.3 / 1 \mathrm{ib}: 1 ; /$ apps/gnu/7.3.0/lib64:1;/apps/gn」

$\hookrightarrow$ u/7.3.0/lib:1;/opt/intel/18.0.3/debugger_2018/li 」

$\hookrightarrow$ bipt/intel64/lib:1;/opt/intel/18.0.3/compilers_a

$\hookrightarrow$ nd_libraries_2018/linux/lib/intel64_lin:1;/opt/i 」

$\hookrightarrow$ ntel/18.0.3/compilers_and_libraries_2018/linux/d 」

$\hookrightarrow$ aal/lib/intel64_lin:1;/opt/intel/18.0.3/compiler

$\hookrightarrow$ S_and_libraries_2018/linux/ipp/lib/intel64_lin:1

$\hookrightarrow$;/opt/intel/18.0.3/compilers_and_libraries_2018/」

$\hookrightarrow$ linux/mkl/lib/intel64_lin:1;/opt/intel/18.0.3/co 」

$\hookrightarrow$ mpilers_and_libraries_2018/linux/tbb/lib/intel64」

$\hookrightarrow$ _lin/gcc4.4:1;/opt/torque/lib64:1;/opt/torque/li 」

$\hookrightarrow \mathrm{b}: 1$

MV2_USE_RDMA_CM=0

_-_Init_Default_Modules $=1$

LMOD_FULL_SETTARG_SUPPORT=no

__LMOD_REF_COUNT_LD_PRELOAD=/apps/xalt/xalt/lib64/li 」

$\hookrightarrow$ bxalt_init.so: 1

LMOD_FAMILY_COMPILER=intel

PBS_NP=28

PBS_NUM_PPN $=28$

QT_PLUGIN_PATH=/usr/lib64/kde4/plugins:/usr/lib/kde4 」

$\hookrightarrow$ /plugins

LMOD_CACHED_LOADS=yes

LMOD_DIR=/apps $/ 1 \mathrm{mod} / 1 \mathrm{mod} / 1 \mathrm{ibexec}$

INCLUDE=/opt/intel/18.0.3/compilers_and_libraries_20 」

$\hookrightarrow$ 18/linux/daal/include:/opt/intel/18.0.3/compiler 」

$\hookrightarrow$ s_and_libraries_2018/linux/ipp/include:/opt/inte 」

$\hookrightarrow \quad 1 / 18.0 .3 /$ compilers_and_libraries_2018/linux/mkl/ 」

$\hookrightarrow$ include 


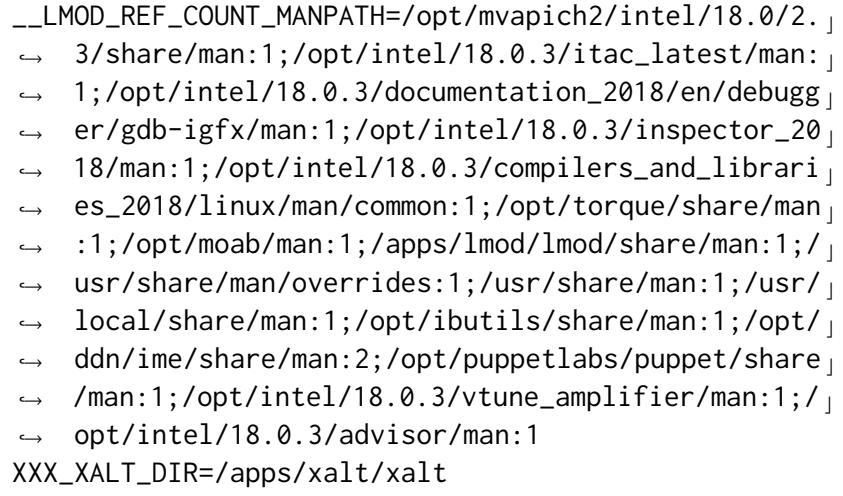

Model:

Model name:

$\hookrightarrow$ a $2.40 \mathrm{GHz}$

Stepping: 4

CPU MHz: $\quad 2400.000$

BogoMIPS: $\quad 4800.00$

Virtualization: $\quad \mathrm{VT}-\mathrm{x}$

L1d cache: $\quad 32 \mathrm{~K}$

L1i cache: $\quad 32 \mathrm{~K}$

L2 cache: $\quad 1024 \mathrm{~K}$

L3 cache: 28160K

NUMA node $0 \mathrm{CPU}(\mathrm{s}): \quad 0,2,4,6,8,10,12,14,16,18,20,22$

$\hookrightarrow \quad, 24,26,28,30,32,34,36,38$

NUMA node1 CPU(s): $\quad 1,3,5,7,9,11,13,15,17,19,21,23$ 」

$\hookrightarrow \quad, 25,27,29,31,33,35,37,39$

Flags: $\quad$ fpu vme de pse tsc msr pae mce

$\hookrightarrow$ cx8 apic sep mtrr pge mca cmov pat pse36 clflush

$\hookrightarrow$ dts acpi mmx fxsr sse sse2 ss ht tm pbe syscall nx

$\hookrightarrow$ pdpe1gb rdtscp lm constant_tsc art arch_perfmon

$\hookrightarrow$ pebs bts rep_good nopl xtopology nonstop_tsc

$\hookrightarrow$ aperfmperf eagerfpu pni pclmulqdq dtes64 monitor

$\hookrightarrow$ ds_cpl vmx smx est tm2 ssse3 sdbg fma cx16 xtpr

$\hookrightarrow$ pdcm pcid dca sse4_1 sse4_2 x2apic movbe popcnt

$\hookrightarrow$ tsc_deadline_timer aes xsave avx $\mathrm{f} 16 \mathrm{c}$ rdrand

$\hookrightarrow$ lahf_lm abm 3dnowprefetch epb cat_13 cdp_13

$\hookrightarrow \quad$ intel_pt ssbd mba ibrs ibpb stibp tpr_shadow vnmi

$\hookrightarrow$ flexpriority ept vpid fsgsbase tsc_adjust bmi1

$\hookrightarrow$ hle avx2 smep bmi2 erms invpcid rtm cqm mpx rdt_a

$\hookrightarrow$ avx512f avx512dq rdseed adx smap clflushopt clwb

$\hookrightarrow \quad$ avx512cd avx512bw avx512vl xsaveopt xsavec

$\hookrightarrow \quad$ xgetbv1 cqm_llc cqm_occup_llc cqm_mbm_total

$\hookrightarrow$ cqm_mbm_local dtherm ida arat pln pts pku ospke

$\hookrightarrow$ spec_ctrl intel_stibp flush_l1d

++ cat /proc/meminfo

MemTotal: $\quad 394800924 \mathrm{kB}$

MemFree: $\quad 379435860 \mathrm{kB}$

MemAvailable: $381470572 \mathrm{kB}$

Buffers: $\quad 208484 \mathrm{kB}$

Cached: $\quad 2844152 \mathrm{kB}$

SwapCached: $\quad 0 \mathrm{kB}$

Active: $\quad 2252220 \mathrm{kB}$

Inactive: $\quad 2267268 \mathrm{kB}$

Active(anon): $1543664 \mathrm{kB}$

Inactive(anon): $\quad 517340 \mathrm{kB}$

Active(file): $\quad 708556 \mathrm{kB}$

Inactive(file): $1749928 \mathrm{kB}$

Unevictable: $\quad 4292464 \mathrm{kB}$

Mlocked: $\quad 4292464 \mathrm{kB}$

SwapTotal: 50331644 kB

SwapFree: $\quad 50331644 \mathrm{kB}$

Dirty: $236 \mathrm{kB}$

Writeback: $0 \mathrm{kB}$

AnonPages: $\quad 5759336 \mathrm{kB}$

Mapped: $\quad 564880 \mathrm{kB}$

Shmem: $\quad 551848$ kB

Slab: $\quad 1804840 \mathrm{kB}$ 


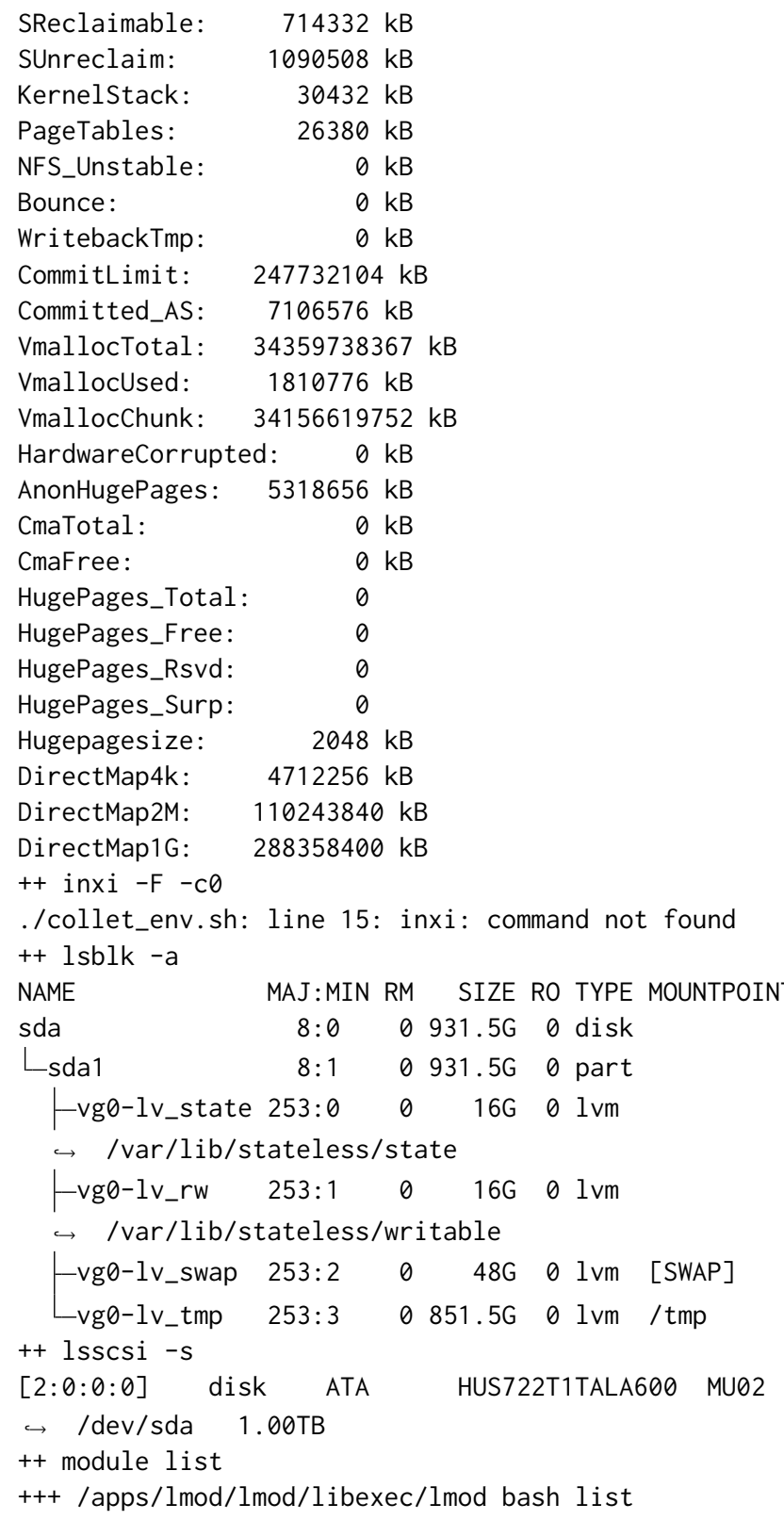

++ eval 'MODULEPATH=/apps/lmodfiles/MPI/intel/18.0/m 」 $\hookrightarrow$ vapich2/2.3:/apps/lmodfiles/Compiler/intel/18.0: 」

$\hookrightarrow /$ apps/lmodfiles/Linux:/apps/lmodfiles/Core:/apps」

$\hookrightarrow / 1 \mathrm{mod} / 1 \mathrm{mod} / \mathrm{modulefiles} /$ Core; ' export

$\hookrightarrow \quad$ 'MODULEPATH;' '_ModuleTable001_=X01vZHVsZVRhYmxl」

$\hookrightarrow \quad X^{\prime}$ X17WyJNVHZlcnNpb24iXTOzLFsiY19yZWJ1aWxkVGltZSJd

$\hookrightarrow$ PTg2NDAwLFsiY19zaG9ydFRpbWUiXT1mYWxzZSxkZXB0aFQ9」

$\hookrightarrow$ e30sZmFtaWx5PXtbImNvbXBpbGVyI109ImludGVsIixbIm1 $\mathrm{w}_{\lrcorner}$

$\hookrightarrow$ aSJdPSJtdmFwaWNoMi IsfSxtVD17Y3h4MTc9e1siZm4iXT0i 」

$\hookrightarrow$ L2FwcHMvbG1vZGZpbGVzLONvbXBpbGVyL2ludGVsLzE4LjAv 」

$\hookrightarrow \quad$ Y3h4MTcvNy4zLjAubHVhIixbImZ1bGxOYW11I109ImN4eDE3 」

$\hookrightarrow \quad$ LzcuMy4wIixbImxvYWRPcmRIciJdPTIscHJvcFQ9e30sWyJz 」

$\hookrightarrow$ dGFja0RlcHRoIl09MixbInN0YXR1cyJdPSJhY3RpdmUiLFsi 」

$\hookrightarrow$ dXNlck5hbWUiXT0iY3h4MTciLH0saW50ZWw9e1 siZm4iXT0i 」

$\hookrightarrow$ L2FwcHMvbG1vZGZpbGVzL0NvcmUvaW50ZWwvMTguMC4zLmx1」

$\hookrightarrow$ YSIsWyJmdWxsTmFt; ' export '_ModuleTable001_;'

$\hookrightarrow$ '_ModuleTable002_=ZSJdPSJpbnRlbC8xOC4wLjMiLFsibG 」

$\hookrightarrow$ 9hZE9yZGVyI109Myxwcm9wVD17fSxbInNOYWNrRGVwdGgiXT」

$\hookrightarrow$ OxLFsic3RhdHVzI109ImFjdGl2ZSIsWyJ1c2VyTmFtZSJdPS ।

$\hookrightarrow$ JpbnRIbCIsfSxtb2R1bGVzPXtbImZuI109Ii9hcHBzL2xtb2」

$\hookrightarrow$ RmaWxlcy9Db3J1L21vZHVsZXMvYXUyMDE4Lmx1YSIsWyJmdW 」

$\hookrightarrow \quad x s T m F t Z S J d P S J t b 2 R 1 b G V L 2 F 1 M j A x O C I s W y J s b 2 F k T 3 J k Z X$ ।

$\hookrightarrow \quad$ IiXT01LHByb3BUPXt9LFsic3RhY2tEZXBOaCJdPTAsWyJzdG

$\hookrightarrow$ FOdXMiXT0iYWNOaXZlIixbInVZZXJOYW11II09Im1vZHVsZX 」

$\hookrightarrow$ MiLHOsbXZhcGljaDI9e1siZm4iXT0iL2FwcHMvbG1vZGZpbG」

$\hookrightarrow \quad$ VzLONvbXBpbGVyL2ludGVsLzE4LjAvbXZhcGljaDIvMi4zLm 」

$\hookrightarrow \quad x 1 Y S I s W y J m d W x s T m F t Z S J d P S J$ tdmFwaWNoMi 8yLjMiLFsibG

$\hookrightarrow$ 9h;' export '_ModuleTable002_;'

$\hookrightarrow$ '_ModuleTable003_=ZE9yZGVyIl09NCxwcm9wVD17fSxbIn 」

$\hookrightarrow \quad$ NOYWNrRGVwdGgiXTOXLFsic3RhdHVzIl09ImFjdGl2ZSIsWy

$\hookrightarrow \quad J 1 c 2$ VyTmFtZSJdPSJtdmFwaWNoMi IsfSx4YWx@PXtbImZuIl

$\hookrightarrow$ 09Ii9hcHBzL2xtb2RmaWxlcy9Db3J1L3hhbHQvbGF0ZXN0Lm」

$\hookrightarrow \quad x 1 Y S I s W y J m d W x s T m F t Z S J d P S J 4 Y W x 0 L 2 x h d G V z d C I s W y J s b 2$ ।

$\hookrightarrow$ FkT3JkZXIiXT0xLHByb3BUPXt9LFsic3RhY2tEZXB0aCJdPT」

$\hookrightarrow$ EsWyJZdGFOdXMiXTOiYWNOaXZlIixbInVZZXJOYW11II09In

$\hookrightarrow$ hhbHQiLHOsfSxtcGFOaEE9eyIvYXBwcy9sbW9kZmlsZXMvTV」

$\hookrightarrow$ BJL2ludGVsLzE4LjAvbXZhcGljaDIvMi4zIiwiL2FwcHMvbG 」

$\hookrightarrow$ 1vZGZpbGVzLONvbXBpbGVyL2ludGVsLzE4LjAiLCIvYXBwcy 」

$\hookrightarrow$ 9sbW9kZmlsZXMvTGludXgiLCIvYXBwcy9sbW9kZmlsZXMvQ2 」

$\hookrightarrow 9 y$;' export '_ModuleTable003_;'

$\hookrightarrow$ '_ModuleTable004_=ZSIsIi9hcHBzL2xtb2QvbG1vZC9tb2 」

$\hookrightarrow$ R1bGVmaWxlcy9Db3J1Iix9LFsic3lzdGVtQmFzZU1QQVRIIl

$\hookrightarrow$ 09Ii9hcHBzL2xtb2RmaWxlcy9MaW51eDovYXBwcy9sbW9kZm」

$\hookrightarrow$ IsZXMvQ29yZTovYXBwcy9sbW9kL2xtb2QvbW9kdWxlZmlsZX 」

$\hookrightarrow \quad M v Q 29 y Z S I s f Q==;$ ' export '_ModuleTable004_;'

$\hookrightarrow$ '_ModuleTable_Sz_=4;' export '_ModuleTable_Sz_;'

+++ MODULEPATH=/apps/lmodfiles/MPI/intel/18.0/mvapic 」

$\hookrightarrow$ h2/2.3:/apps/lmodfiles/Compiler/intel/18.0:/apps」

$\hookrightarrow /$ Imodfiles/Linux:/apps/lmodfiles/Core:/apps/lmod」

$\hookrightarrow / 1 \mathrm{mod} / \mathrm{modulefiles/Core}$

+++ export MODULEPATH 


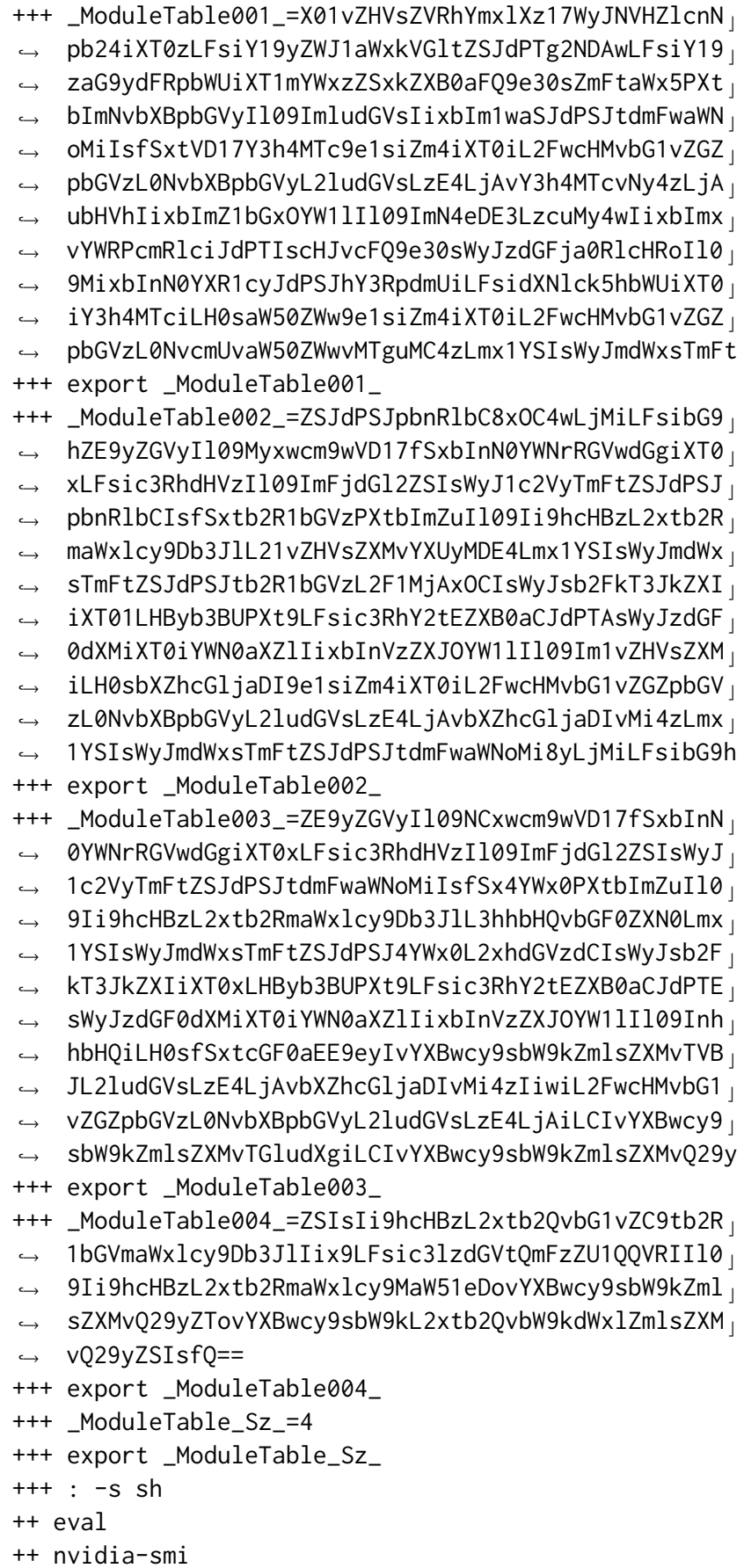

Thu Apr 11 06:33:06 2019

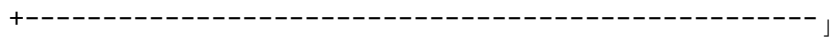

$\hookrightarrow--------------------------+$

I NVIDIA-SMI 410.79 Driver Version: 410.79

$\hookrightarrow$ CUDA Version: $10.0 \quad$ ।

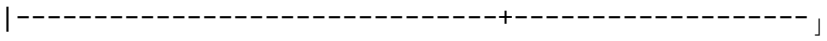

$\hookrightarrow---+----------------------+$

| GPU Name Persistence-M| Bus-Id Disp.A

$\hookrightarrow$ | Volatile Uncorr. ECC |

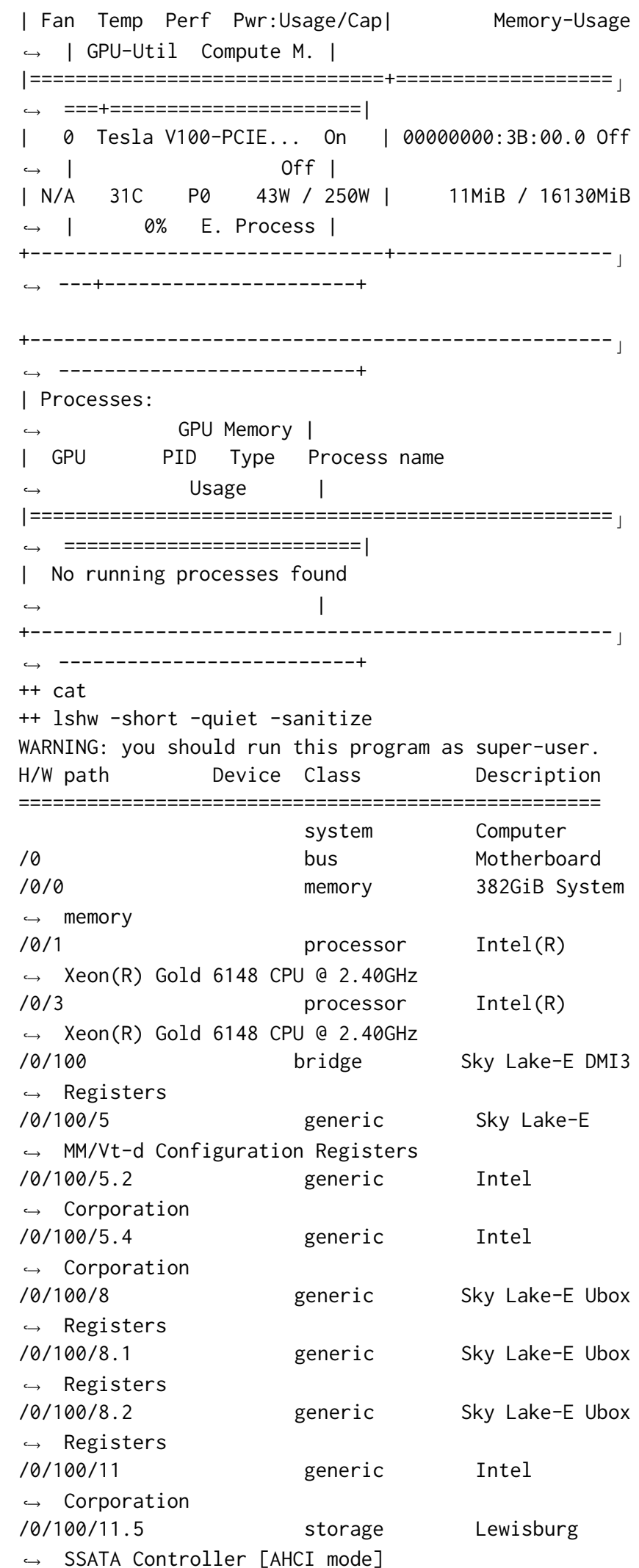

\section{$\hookrightarrow$ | GPU-Util Compute M. |}

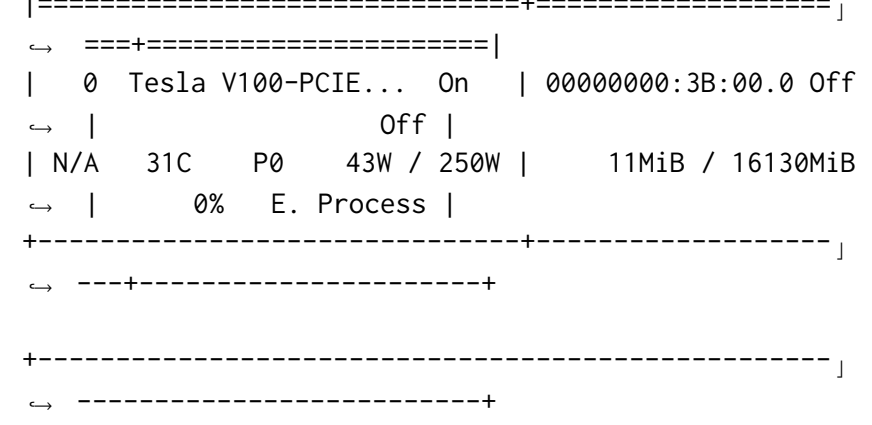

| Processes:
$\hookrightarrow \quad$ GPU Memory ।
$\hookrightarrow \quad$ Usage |

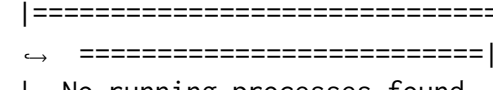

| No running processes found

$$
\hookrightarrow
$$

++ lshw -short -quiet -sanitize

WARNING: you should run this program as super-user.

$\mathrm{H} / \mathrm{W}$ path Device Class Description

/0/100 bridge

$\hookrightarrow$ Registers

10/100/5 generic

$\hookrightarrow \mathrm{MM} / \mathrm{Vt}-\mathrm{d}$ Configuration Registers

/0/100/5.2 generic

$\hookrightarrow$ Corporation

$10 / 100 / 5.4$

$\hookrightarrow$ Corporation

$\hookrightarrow$ Registers

/0/100/8.1

$\hookrightarrow$ Registers

10/100/8.2

$\hookrightarrow$ Registers

$\hookrightarrow$ Corporation

$\hookrightarrow$ SSATA Controller [AHCI mode] 
Nisa, et al.

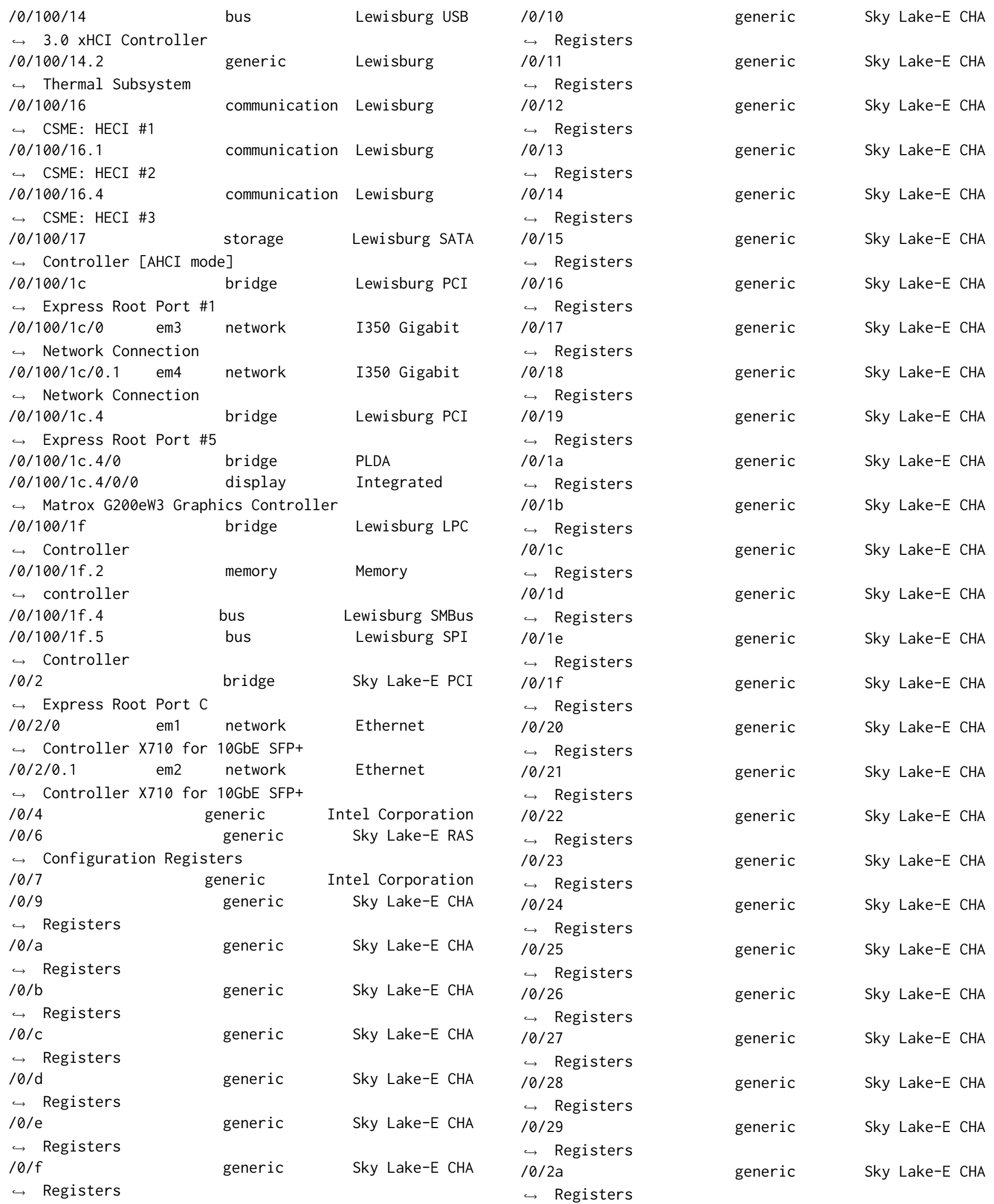


An Efficient Mixed-Mode Representation of Sparse Tensors

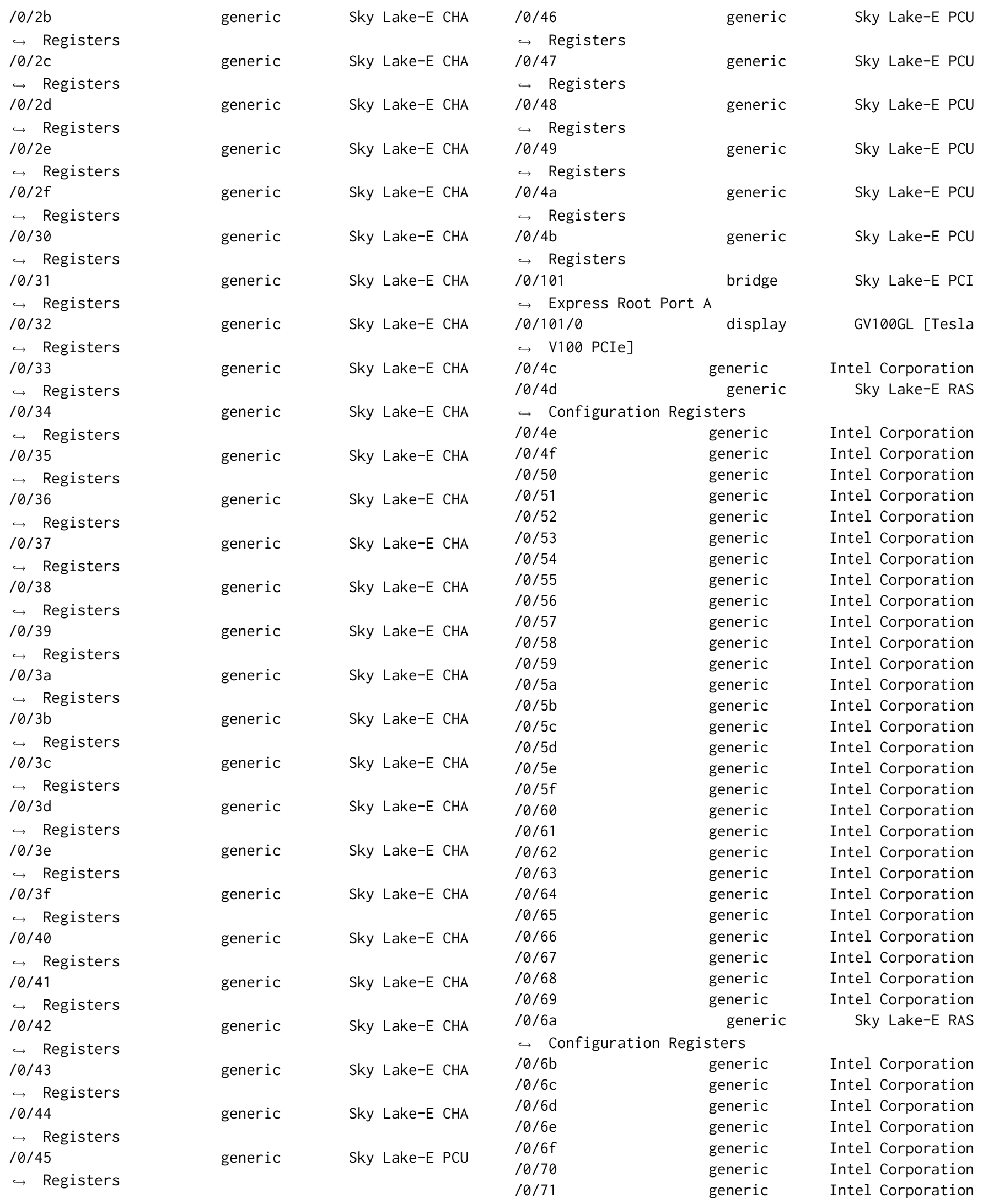


Nisa, et al.

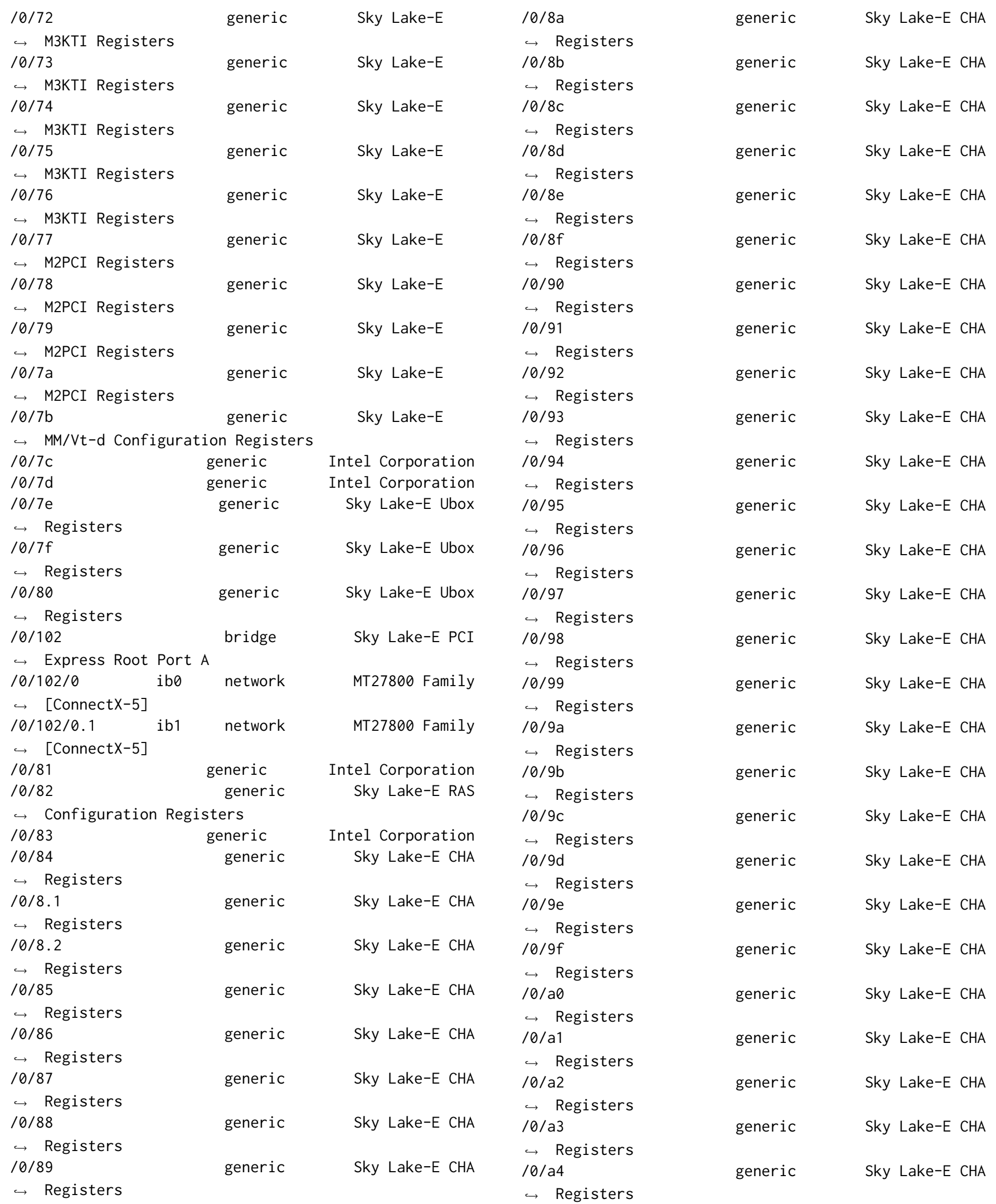


An Efficient Mixed-Mode Representation of Sparse Tensors

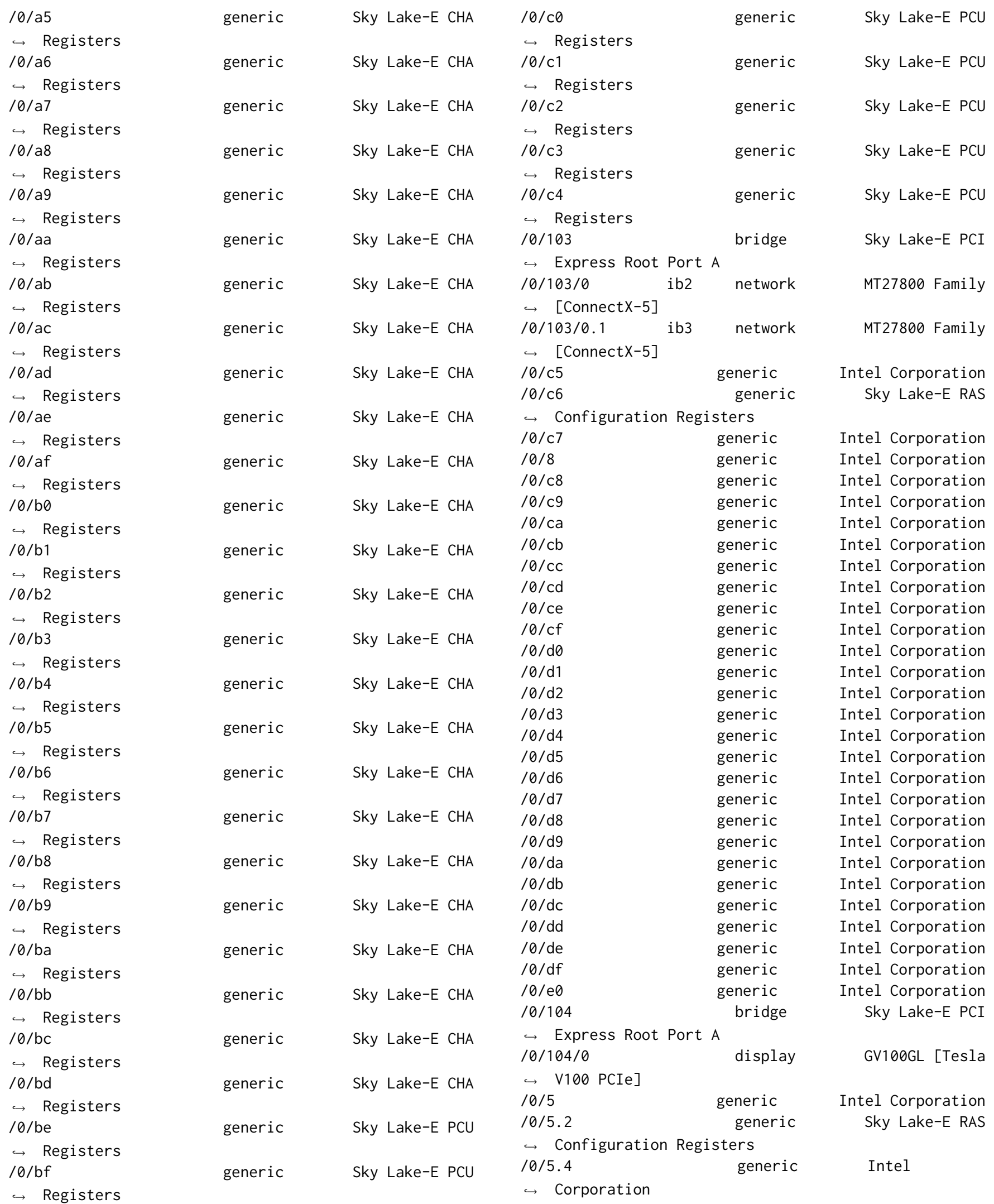




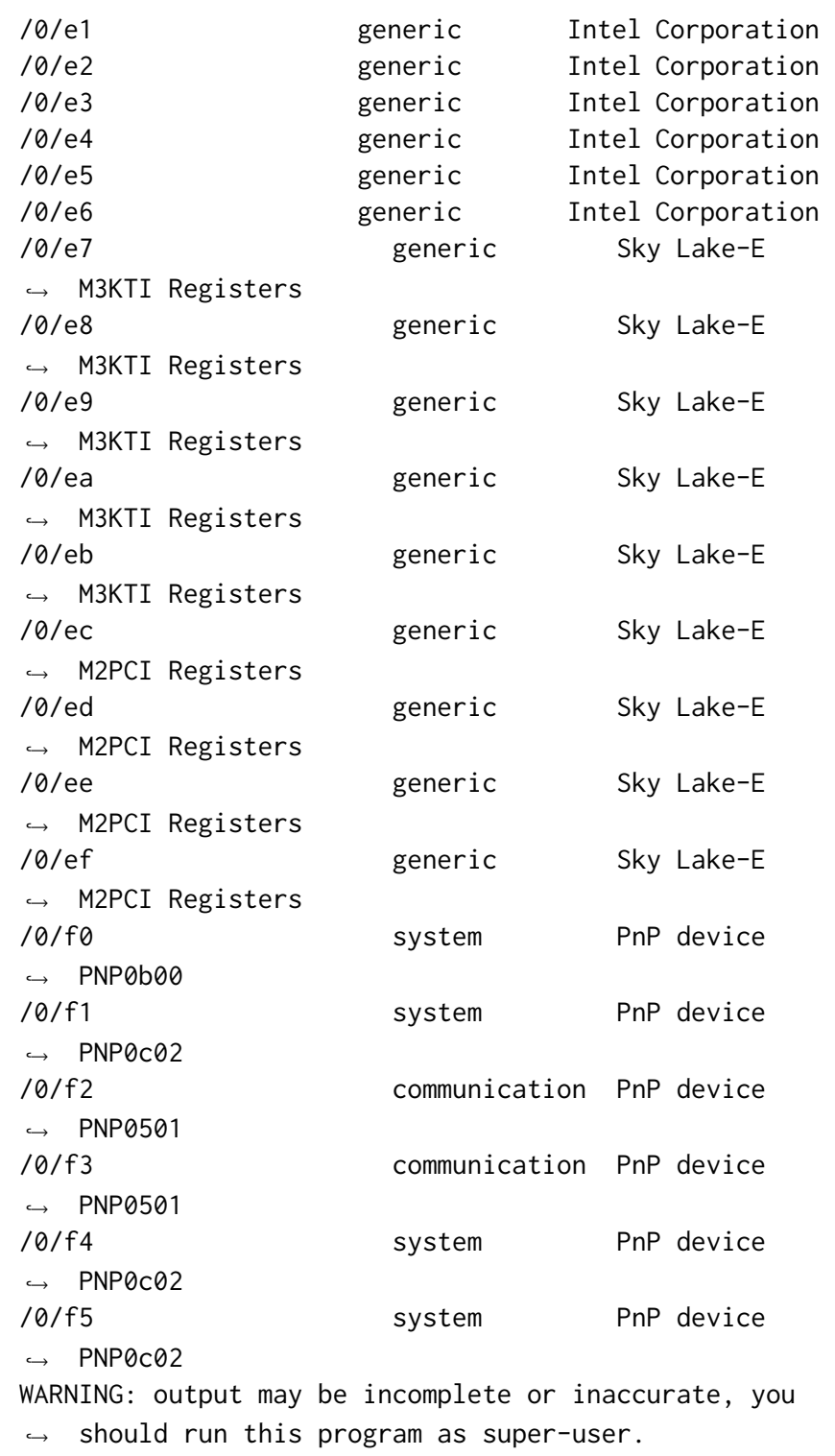

\section{ARTIFACT EVALUATION}

Verification and validation studies: We evaluate the performance of the proposed MM-CSF format against six benchmarks. Among these six frameworks, BCSF-ALL, ParTI-COO, and FCOO are GPU based frameworks. HiCOO, SPLATT-ALL and SPLATT-ONE are CPU based frameworks. We show performance in terms of GFLOPS on six datasets: deli, nell1, nell2, flick, fr_m, fr_s and darpa. The steps to collect datasets and the running procedure can be found in the repository. We expect MM-CSF to outperform all other benchmarks for all the cases.

Accuracy and precision of timings: The following two tables summarize the GFLOPS achieved by MM-CSF and other state-of-the-art benchmarks on CPUs and GPUs.The column header represents the name of the benchmarks. The row header represents the datasets.

GFLOPS of MM-CSF and other GPU based frameworks:
MM-CSF, ALL-BCSF, ParTI-COO, FCOO deli: 364, 333, 271, fails nell1: 285, 270, 176, fails nell2: 763, 607, 313, fails flick: 435, 327, 295 ,fails fr_m: 235, 194, 127, fails fr_s: 228, 203, fails, fails darpa: $327,209,100,29$

GFLOPS of MM-CSF and other CPU based frameworks:

MM-CSF, HiCOO, SPLATT-ALL, SPLATT-ONE deli: 364, 7, 8, 13 nell1: 285, 5, 17, 18 nell2: 763, 78, 150, 225 flick: 435, 4, 7, 25 fr_m: 235, 5, 5, 4 fr_s: 228, 5, 4, 4 darpa: 327, 7, 7, 1 